\title{
Average density estimation for urban traffic networks: application to the Grenoble network
}

\author{
Martin Rodriguez-Vega ${ }^{\mathrm{a}, *}$, Carlos Canudas-de-Wit ${ }^{\mathrm{a}}$, Hassen Fourati ${ }^{\mathrm{a}}$ \\ ${ }^{a}$ CNRS, Gipsa-Lab, Grenoble INP, Univ. Grenoble Alpes, INRIA, 38000 Grenoble, France
}

\begin{abstract}
This paper deals with the problem of the average density estimation in largescale traffic networks, without requiring to know the density of each individual road. This is achieved by the design of a reduced-order open-loop observer. In general, this type of observers require some specific graph properties, but we show that it is possible to find a virtual representation of the network that satisfies these conditions, by diving each road into a number of cells of specific length. The virtual network is shown to provide close approximations to the average density of the real system for large enough networks. Algorithms to efficiently calculate the observer parameters are proposed. This approach is based on the assumption that traffic dynamics are linear, and is applied at first in free-flow regime. Using microscopic simulations and real data we evaluate the observer performance even when congestion is present in the network.
\end{abstract}

Keywords: Average density estimation, Large traffic networks, Virtual divisions, Open-loop observer

\section{Introduction}

Traffic state estimation (TSE) is important in modern Intelligent Transportation Systems (ITS) and refers to the process of observation of traffic state variables (not observed everywhere in the network), such as flow, density, speed, and other equivalent variables, on road segments, using partially observed and noisy traffic data. For example, precise TSE is required usually in ramp metering, pricing, and information provision in order to mitigate congestion effectively. For this purpose, different sensors such as loop detectors, radars, magnetometers, etc. are used to collect the traffic measurements. The goal with estimation algorithms is to observe the whole network's traffic states.

First proposed works with classical TSE were applied to highways. Tampère and Immers (2007) proposes the use of an Extended Kalman Filter (EKF) coupled with the well known Cell Transmission Model (CTM) to estimate the density of sections of highways, using data from stationary sensors located at certain

*Corresponding author. E-mail address: Martin.Rodriguez-Vega@gipsa-lab.fr 
sections of the road. This approach is based on linearlizing the CTM around a current state, and then compare the measurements with the predictions of the linearized model. Later, Herrera and Bayen (2010) made use of the increase of availability of GPS data to complement the stationary sensor data. In this formulation, a Lagrangian model was used as input to the Kalman filter. More recently, other sources of information such as connected vehicles have been used, Seo et al. (2015a). Connected vehicles are provided with sensors capable of measuring its surrounding, such as the inter-vehicle distances and speeds. This information is then transmitted to a centralized location. Sun and Work (2014); Seo et al. (2015b) show how this information can be provided to a Kalman filtering approach to improve highway state reconstruction.

More and more, the need to study urban traffic networks is increasing in the last years for TSE issues. Some methods have been developed to estimate traffic states such as Unscented Kalman Filter (UKF) in Pueboobpaphan and Nakatsuji (2006), and Bayesian probabilistic model-based Expectation-Maximization Extended Kalman Filter (EM-EKF) in Gu et al. (2017). Nevertheless, in comparison to highways, urban networks still have not received as much attention, Seo et al. (2017). This is due in part to the complexity of modeling traffic dynamics with intersections, the related entry and exit flows, and the lack of sufficient sensor data. For instance, in this context, the main focus was on determining the optimal number of sensors and their locations to estimate the states of the network. $\mathrm{Ng}$ (2012) proposes a method to identify the minimum number of stationary sensors required to estimate the flow of all roads in the network. This is done by expressing flow conservation laws at each node as a set of linear equations, and then completing the rank of the resulting matrix using the Gaussian algorithm. Later, He (2013) proposes a graph-based approach to identify the optimal sensor locations in a more computational efficient manner. This work also provides an algorithm to estimate the equilibrium flow for each road from sensor data using also graph-based algorithms. In Lovisari et al. (2016), a method to estimate the dynamical evolution of the density of each road is proposed by using both stationary sensor measurements and Floating Car Data (FCD). This is done by first giving a flow estimate for each road as if the network is in stead-state, and then use the speed-density fundamental diagram to provide density pseudo-measurements. This estimates are then the input to a Luenberger-like observer. In Ladino et al. (2018), FCD is used to determine if each road is in free-flow or congested regime, and then uses the flow-density fundamental diagram and stationary sensor data to provided density estimates to each road in the traffic network. More recently, Rostami Shahrbabaki et al. (2020) proposes a data-based density estimation method that does not require the use of a fundamental diagram. Instead, data from connected vehicles is used to estimate the outflow of each road, and then uses the density conservation law to estimate the traffic state. However, as these methods provide density and flow values for each road, as the size of the network increases the required number of computations increases as well. This might be too computationally expensive and sometimes we do not dispose of the sufficient number of sensors to install in each road of the network. Also, it looks that in some roads of certain 
neighborhoods in urban traffic networks, cars rarely pass, then it might not be necessary to estimate the density in each of such roads.

The alternative to these methods in that case is to partition the network into regions (groups of interconnected roads) and estimate their average states. Some works are conducted in that direction. In Geroliminis and Daganzo (2008), the experimental evidence of a Macroscopic Fundamental Diagram (MFD) was obtained, which is a relationship between the average density of a region with its outgoing flow. Haddad and Geroliminis (2012) studies the stability of traffic control for two-region urban cities. A city is partitioned in two regions with a MFD for each of the regions. A set of conditions are derived for stable equilibrium accumulations in the undersaturated regimes for both MFDs. Moreover, the traffic perimeter control problem for the two-region MFDs system is formulated. Aboudolas and Geroliminis (2013) generalizes this control technique to any number of regions. Haddad and Zheng (2020) also studies the use of the MFD for multi-region perimeter control, but by modifying the MFD as to include time-delays between the changes in the inputs and the observed outputs. In other applications, Saedi et al. (2020) estimates the traveling time between two points, by dividing an heterogeneous network into homogeneous sections, each with a well-defined MFD. Aboudolas and Geroliminis (2013) and Saedi et al. (2020) use limited measurements of density in certain roads, which is then used as a proxy for the entire region. This may incur in an error in the inputs of the MFD. It is to note that in these approaches, the average-density estimation per region is too approximate and we find the precision questionable.

By exploring last researches on network theory, it looks that few recent works start to focus on the estimation of average states using low dimensional observers for large-scale network systems as in Sadamoto et al. (2017) and Niazi et al. (2019). The established conditions for the existence of such observers result in the concept of average detectability. Based on these works, we proposed in this paper to explore the applicability of such theory to traffic network when sensors (e.g. magnetic loop detectors) are available only at the boundaries and estimate the average density of a predefined region. This will represent our main contribution in the area of urban traffic networks and can be the main work treating this issue to the best of the author's knowledge. Due to the complexity of modeling traffic dynamics with intersections, we assume that traffic networks follow linear dynamics and focus at first on free-flow regime. This work remains important for region-based estimators where free-flow conditions are satisfied (interconnected residential neighborhoods or regions of cities with low/moderate traffic for examples). Moreover, if the region does not satisfy the linear dynamics condition, the proposed estimator can still provide bounds on the average density. Even though the underlying methods assume free-flow, simulations with congested regimes are carried out to model the resulting error, and to provide working ranges where the estimator is applicable under mixed regimes. Finally, such proposed theory can prepare for future work focusing on the same problem but under high congestion.

The paper is organized as follows: in Section 2 we introduce the model for traffic state evolution and the conditions required for the existence of an ob- 
server for the average density. Section 3 shows simple motivating examples that suggest how choosing specific road divisions yields average detectable networks. Section 4 formally states the definition of a virtual network and its average density observer. Section 5 presents the main results on how to select road divisions for general networks. Section 6 analyses the error induced by the observer. Section 7 demonstrates the use of the proposed methods on a Manhattan grid. Finally, Section 8 describes the use of real data from a region of the city of Grenoble in France, to validate the proposed techniques.

\section{Background}

\subsection{Traffic model}

In this paper, we model traffic networks as a direct graph $\mathcal{G}=\{\mathcal{N}, \mathcal{E}, R\}$, where the nodes $\mathcal{N}=\{1,2, \ldots, p\}$ represent sections of roads, the edges $\mathcal{E} \subset$ $\mathcal{N} \times \mathcal{N}$ represent the possible vehicle transfers (turns) from one road to another, and $R \in \mathbb{R}^{p \times p}$ is the adjacency matrix whose elements $r_{i, j}$ (also called turning or split ratios) are the fraction of vehicles that turn from road $i$ to road $j$. In addition to $\mathcal{G}$, we include a set of parameters for each road representing physical properties, e.g., a vector of road lengths $\boldsymbol{\ell} \in \mathbb{R}^{p}$.

The traffic dynamics for a given graph defines the trajectories of the density $\boldsymbol{\rho}(t)$, the incoming (or upstream) flow $\boldsymbol{\varphi}_{\text {in }}(t)$, and the outgoing (or downstream) flow $\varphi_{\text {out }}(t)$, for each road at every time $t$. The dynamics are obtained as in Bianchin et al. (2019) from the conservation law

$$
\dot{\boldsymbol{\rho}}(t)=L^{-1}\left(\boldsymbol{\varphi}_{\text {in }}(t)-\boldsymbol{\varphi}_{\text {out }}(t)\right)
$$

where $L=\operatorname{diag}(\ell)$.

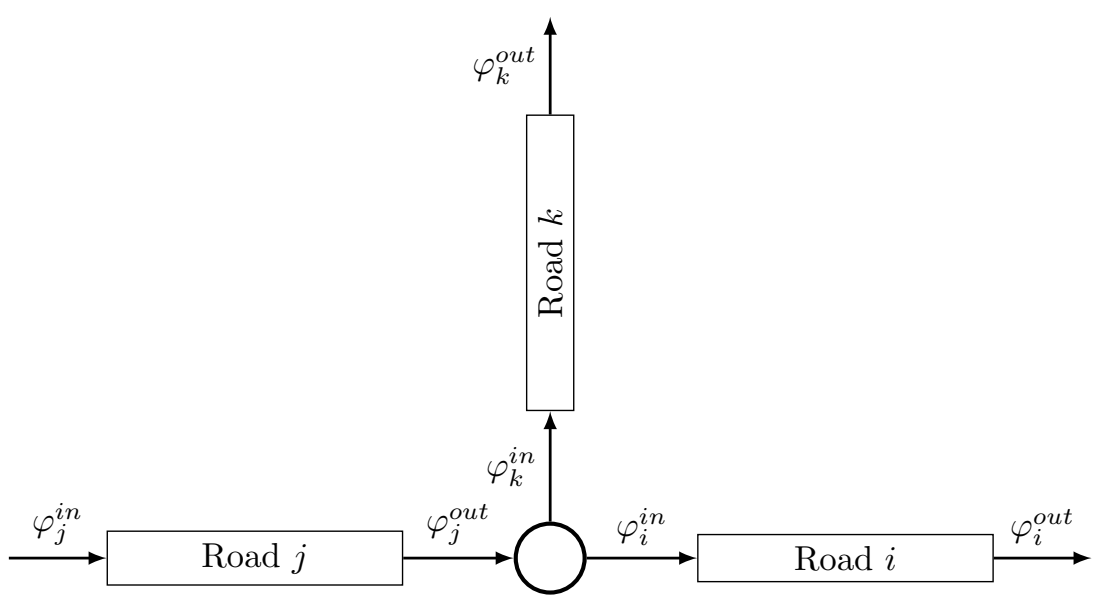

Figure 1: Flow exchange at an intersection. 
As shown in Fig. 1, the incoming flow for each road is equal to the sum of the outgoing flows of the upstream roads, weighted by the corresponding turning ratio,

$$
\varphi_{i}^{\text {in }}(t)=\sum_{j \neq i} r_{j, i} \varphi_{j}^{\text {out }}(t) .
$$

Taking into account all roads, this can be written in matrix notation as

$$
\boldsymbol{\varphi}_{\text {in }}(t)=R^{\top} \boldsymbol{\varphi}_{\text {out }}(t)+B \mathbf{u}(t)
$$

where $B \in\{0,1\}^{p \times q}$ is a selection matrix that indicates the location of boundary inflows, and $\mathbf{u}(t) \in \mathbb{R}^{q}$ contains the input demands. Thus, (1) becomes,

$$
\dot{\boldsymbol{\rho}}(t)=L^{-1}\left(R^{\top}-\mathbb{I}\right) \boldsymbol{\varphi}_{\text {out }}(t)+L^{-1} B \mathbf{u}(t) .
$$

To calculate the output flow $\varphi_{\text {out }}(t)$ we use the well known Cell Transmission Model (CTM) first proposed in Daganzo (1994) and Daganzo (1995). For a single road this model makes use of the triangular Fundamental diagram, which expresses the flow as a function of the density as shown in Fig. 2. This Fundamental Diagram is a piecewise linear function with a single breakpoint, whose parameters are the free-flow speed $v$, the backward wave speed $w$, the capacity $\varphi_{\max }$, and the jam density $\rho_{\max }$. In matrix notation, we define $\left\{\mathbf{v}, \mathbf{w}, \boldsymbol{\varphi}_{\max }, \boldsymbol{\rho}_{\max }\right\} \subset \mathbb{R}^{p}$ as the vectors of the corresponding parameters for all roads. The density coordinate of the breakpoint is called the critical density $\rho_{c}$, and is directly calculated from the other parameters. A road whose density is less than the critical value is said to be in free-flow. Conversely, if the density is greater than $\rho_{c}$, the road is said to be congested.

When considering a network of roads, the effect of adjacent nodes has to be taken into consideration. Define the Demand $D(\boldsymbol{\rho}(t))$ and Supply $S(\boldsymbol{\rho}(t))$ functions as the maximum flow of vehicles that can leave and enter each road at time $t$, respectively. These are evaluated as

$$
D(\boldsymbol{\rho})=\min \left\{V \boldsymbol{\rho}, \boldsymbol{\varphi}_{\max }\right\} \quad, \quad S(\boldsymbol{\rho})=\min \left\{W\left(\boldsymbol{\rho}_{\max }-\boldsymbol{\rho}\right), \boldsymbol{\varphi}_{\max }\right\}
$$

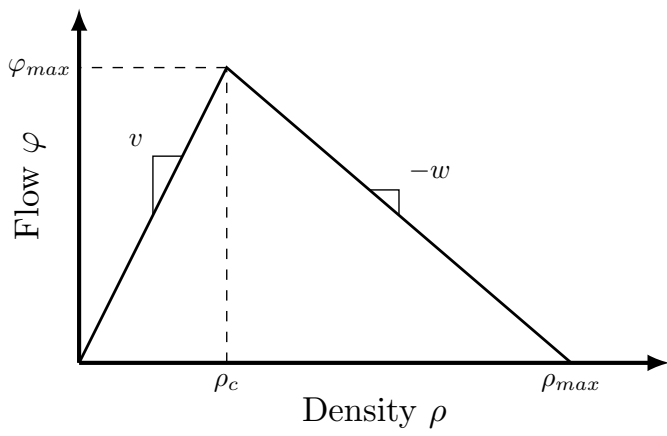

Figure 2: Triangular fundamental diagram. 
where $V=\operatorname{diag}(\mathbf{v})$ and $W=\operatorname{diag}(\mathbf{w})$.

With these definitions, the outgoing flow for each cell is given by the optimization problem,

$$
\begin{aligned}
\boldsymbol{\varphi}_{\text {out }}(t)=\underset{\boldsymbol{a}}{\operatorname{argmax}} & \mathbf{1}^{\top} \boldsymbol{\varphi} \\
\text { s.t. } & \left\{\begin{aligned}
\boldsymbol{\varphi} & <D(\boldsymbol{\rho}(t)), \\
R^{\top} \boldsymbol{\varphi} & <S(\boldsymbol{\rho}(t)), \\
0 & <\boldsymbol{\varphi} .
\end{aligned}\right.
\end{aligned}
$$

This is called the flow-maximizing junction model, as it assumes that the throughput of each intersection is always maximized. This is the most common choice of model, but other options are available, see Jabari (2016).

\subsubsection{Linear dynamics}

In this paper, we examine the convergence of a one-dimensional estimator for the average density of the whole network. However, due to the limitations of the available tools to analyze the observability and detectability of non-linear systems, we are restricted to the case of linear systems of the form

$$
\dot{\boldsymbol{\rho}}(t)=A \boldsymbol{\rho}(t)+L^{-1} B \mathbf{u}(t)
$$

where $A=L^{-1}\left(R^{\top}-\mathbb{I}\right) V$ is a time-invariant matrix. Note than (7) implies that the velocity of each road $V$ is constant and independent of the density $\boldsymbol{\rho}$. This limits the working range of our proposed method, as it is clear that the CTM given dynamics in (6) are non-linear, as the velocity of vehicles decreases with increasing density. For instance, the case when all roads are in free-flow, $\boldsymbol{\rho} \leq \boldsymbol{\rho}_{c}$, satisfies the required condition, but in the congested regime, the road outflow decreases as the density increases, implying the average vehicle speed decreases as well.

Despite this limitation, Section 8 evaluates the performance of the estimator under congested scenarios. It was found that for some cases, even multiple congestions in a considered region still provide an error under $10 \%$. Nevertheless, the extension of this work to consider more general cases with congestion is a work in progress.

\subsection{Average density dynamics}

Consider that sensors are located in a set of nodes $\mathcal{S} \subset \mathcal{N}$ corresponding to the boundaries (inflows and outflows) of the network. Without loss of generality, we index roads such that measured roads have the highest indexes, i.e., $\mathcal{S}=$ $\{p-s+1, \ldots, p\}$ with $q<s<p$. Thus, $\mathbf{y}(t)=C \boldsymbol{\rho}(t)$ where $C=\left[\begin{array}{ll}\mathbf{0}_{s \times m} & \mathbb{I}_{s}\end{array}\right]$, and $m=p-s$ is the number of unmeasured nodes.

Consider a partition of the state vector as $\boldsymbol{\rho}(t)=\left[\begin{array}{ll}\boldsymbol{\rho}_{1}^{\top}(t) & \boldsymbol{\rho}_{2}^{\top}(t)\end{array}\right]^{\top}$ such that $\boldsymbol{\rho}_{1}(t) \in \mathbb{R}^{m}$ corresponds to the states of the unmeasured nodes, and $\boldsymbol{\rho}_{2}(t) \in \mathbb{R}^{s}$ to the states of the measured nodes. Note that $\boldsymbol{\rho}_{2}(t)=\mathbf{y}(t)$. The system matrices are partitioned accordingly,

$$
A=\left[\begin{array}{ll}
A_{11} & A_{12} \\
A_{21} & A_{22}
\end{array}\right], \quad B=\left[\begin{array}{l}
B_{1} \\
B_{2}
\end{array}\right] .
$$


where $A_{11} \in \mathbb{R}^{m \times m}, A_{12} \in \mathbb{R}^{m \times s}, A_{21} \in \mathbb{R}^{s \times m}, A_{22} \in \mathbb{R}^{s \times s}$, and $B_{1} \in \mathbb{R}^{m \times q}$ and $B_{2} \in \mathbb{R}^{s \times q}$. Analogously, let

$$
R=\left[\begin{array}{ll}
R_{11} & R_{12} \\
R_{21} & R_{22}
\end{array}\right], L=\left[\begin{array}{cc}
L_{1} & \mathbf{0} \\
\mathbf{0} & L_{2}
\end{array}\right], V=\left[\begin{array}{cc}
V_{1} & \mathbf{0} \\
\mathbf{0} & V_{2}
\end{array}\right] .
$$

We aim to estimate the average of the unmeasured states, i.e., $\rho_{a v}(t)=$ $\frac{1}{m} \mathbf{1}^{\top} \boldsymbol{\rho}_{1}(t)$ without requiring knowledge about the full vector $\boldsymbol{\rho}_{1}(t)$. Consider a lower-dimensional projected system in which the unmeasured states are aggregated. The average state follows

$$
\dot{\rho}_{a v}(t)=\frac{1}{m} \mathbf{1}^{\top} A_{11} \mathbf{1} \rho_{a v}(t)+\frac{1}{m} \mathbf{1}^{\top} A_{12} \boldsymbol{\rho}_{2}(t)+\frac{1}{m} \mathbf{1}^{\top} A_{11} \boldsymbol{\sigma}(t)+\frac{1}{m} \mathbf{1}^{\top} B_{1} \mathbf{u}(t)
$$

where $\boldsymbol{\sigma}(t)$ is the average deviation vector given by $\boldsymbol{\sigma}(t)=\boldsymbol{\rho}_{1}(t)-\mathbf{1} \rho_{a v}(t)$.

\subsection{Average detectability}

Consider the open-loop observer

$$
\dot{\hat{\rho}}_{a v}(t)=\frac{1}{m}\left[\mathbf{1}^{\top} A_{11} \mathbf{1} \hat{\rho}_{a v}(t)+\mathbf{1}^{\top} A_{12} \mathbf{y}(t)+\mathbf{1}^{\top} B_{1} \mathbf{u}(t)\right]
$$

which is obtained by following the known dynamics (10) ignoring the unknown input $\boldsymbol{\sigma}(t)$.

Definition: a system is called average detectable if the open-loop observer (11) converges asymptotically, i.e., $\hat{\rho}_{a v}(t) \rightarrow \rho_{a v}(t)$ as $t \rightarrow \infty$.

Note that average detectability is a particular case of Functional Observability, as described in Fernando et al. (2010). The following theorem can determine if a given network is average detectable based on the structure of the transition matrix,

Theorem 1. [Niazi et al. (2019)] For systems of the form (10), the open-loop observer (11) converges if and only if $\mathbf{1}^{\top} A_{11}=-\gamma \mathbf{1}^{\top}$ with $\gamma>0$.

In the following section we show that these conditions are not generally satisfied for traffic networks. Nevertheless, we will show by dividing each road into virtual cells it is possible to construct networks which are average detectable.

\section{Motivating examples}

\subsection{One way road}

Consider a one way road as shown in Fig. 3.a. Sensors are located at the upstream and downstream boundaries of the road, represented by green strips in the figure. Let $\ell$ be the length of road between the sensors, and $v$ be the maximum velocity. We divide this stretch into 3 virtual sections (cells), such that the sum of their lengths is $\ell$, and all of them have maximum velocity $v$. Possible divisions are shown in Figs. 3.b and 3.c. 


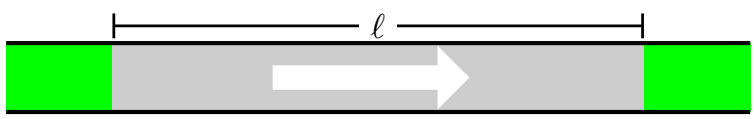

(a) One way road

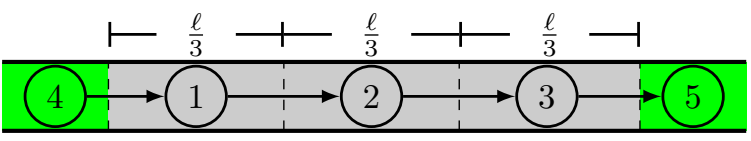

(b) Homogeneous cells

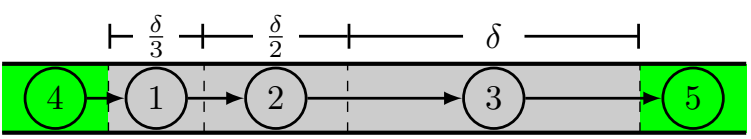

(c) Inhomogeneous cells

Figure 3: One way road. Green strips represent sensors located and the upstream and downstream ends. Two different virtual divisions are shown.

First, consider the common approach of considering homogeneous cells as in Fig. 3.b, such that cells 1-3 have each length $\ell / 3$. The corresponding state matrix is,

$$
A=\left[\begin{array}{ccc|cc}
-3 \ell^{-1} & 0 & 0 & 3 \ell^{-1} & 0 \\
3 \ell^{-1} & -3 \ell^{-1} & 0 & 0 & 0 \\
0 & 3 \ell^{-1} & -3 \ell^{-1} & 0 & 0 \\
\hline 0 & 0 & 0 & -\ell_{4}^{-1} & 0 \\
0 & 0 & \ell_{5}^{-1} & 0 & -\ell_{5}^{-1}
\end{array}\right] v
$$

where $\ell_{4}$ is the length of entry and $\ell_{5}$ is the length of the exit. It can be seen that $\mathbf{1}^{\top} A_{11}=\left[\begin{array}{lll}0 & 0 & -3 \ell^{-1}\end{array}\right] v$. Thus, the condition $\mathbf{1}^{\top} A_{11}=-\gamma \mathbf{1}^{\top}$ from Theorem 1 is not satisfied, and thus, equal length divisions are not average detectable.

This results seems counterintuitive, as it is known that for one ways roads such as highways, measuring the density of the downstream cell is enough to make the entire system observable and the density of each cell can be known, Bekiaris-Liberis et al. (2016). However, we are interested in estimating the average density of all cells directly, without the need to calculate each individual density. Therefore, full observability does not imply average detectability.

Now, consider a division such that cell 3 has length $\delta$, cell 2 has length $\delta / 2$ and cell 1 has length $\delta / 3$, where $\delta=\frac{6}{11} \ell$ (see Fig. 3.c). The corresponding state matrix is

$$
A=\left[\begin{array}{ccc|cc}
-3 \delta^{-1} & 0 & 0 & 3 \delta^{-1} & 0 \\
2 \delta^{-1} & -2 \delta^{-1} & 0 & 0 & 0 \\
0 & \delta^{-1} & -\delta^{-1} & 0 & 0 \\
\hline 0 & 0 & 0 & -\ell_{4}^{-1} & 0 \\
0 & 0 & \ell_{5}^{-1} & 0 & -\ell_{5}^{-1}
\end{array}\right] v
$$




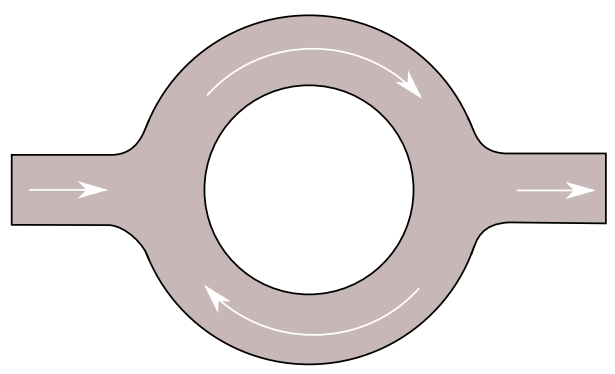

Figure 4: Circle road with one entry and one exit.

and thus $\mathbf{1}^{\top} A_{11}=\left[\begin{array}{lll}-\delta^{-1} & -\delta^{-1} & -\delta^{-1}\end{array}\right] v$. Note that all column sums are equal, and because of Theorem 1 , this division is average detectable.

\subsection{Circle road: networks}

Consider a ring road as shown in Fig. 4. Suppose that sensors are located at the entry and the exit. Consider the network representation in Fig. 5. The green nodes represent sensors in the network boundaries. For simplicity, we no longer index the nodes with sensors as they are not concerned with the average detectability conditions.

Denote by $\ell_{1}, \ell_{2}, v_{1}, v_{2}$ the lengths and free-flow velocities of the top and bottom sections of the circle, respectively. In this example, we are interested in expressing the required conditions for the lengths, so to simplify the writing of the equations let $v_{1}=v_{2}=1$. The state matrix of the unmeasured partition is

$$
A_{11}=\left[\begin{array}{cc}
-\ell_{1}^{-1} & \ell_{1}^{-1} \\
r \ell_{2}^{-1} & -\ell_{2}^{-1}
\end{array}\right]
$$

According to Theorem 1, to be able to reconstruct the average density this matrix must satisfy $\mathbf{1}^{\top} A_{11}=-\gamma \mathbf{1}^{\top}$, and therefore

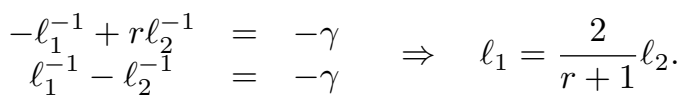

As $r<1$, the roads cannot be of equal length. Thus, we are interested in finding a way to modify the network, such that physical parameters are conserved (i.e. lengths, velocities and turning ratios), but that the network is average detectable.

Let the physical lengths of the roads 1 and 2 be $\ell_{1}=\ell_{2}=\ell$. Consider a new network where roads 1 and 2 are divided into $n_{1}$ and $n_{2}$ cells, respectively, as shown in Fig. 6. Let cells 1 to $n_{1}$ correspond to road 1 , and cells $n_{1}+1$ to $n_{1}+n_{2}$ correspond to road 2. Furthermore, let $\delta_{i}$ be the length of the $i$-th cell. The dimension and elements of this network's state matrix, denoted by 
$A^{\left(n_{1}, n_{2}\right)}$, depend on the values of $n_{1}, n_{2}$ and the vector of cell lengths $\boldsymbol{\delta}$. The block matrix corresponding to the unmeasured states is

$$
A_{11}^{\left(n_{1}, n_{2}\right)}=\left[\begin{array}{ccccccc}
-\delta_{1}^{-1} & \delta_{1}^{-1} & 0 & \cdots & 0 & 0 & 0 \\
0 & -\delta_{2}^{-1} & \delta_{2}^{-1} & \cdots & 0 & 0 & 0 \\
\vdots & & \ddots & & & & \vdots \\
0 & \cdots & & -\delta_{n_{1}}^{-1} & \delta_{n_{1}}^{-1} & \cdots & 0 \\
0 & \cdots & & 0 & -\delta_{n_{1}+1}^{-1} & \cdots & 0 \\
\vdots & & & & & \ddots & \vdots \\
r \delta_{n_{1}+n_{2}}^{-1} & 0 & \cdots & 0 & 0 & \cdots & -\delta_{n_{1}+n_{2}}^{-1}
\end{array}\right] .
$$

The average detectability condition requires $-\delta_{1}^{-1}+r \delta_{n_{1}+n_{2}}^{-1}=-\gamma$ and $-\delta_{i}^{-1}+$ $\delta_{i-1}^{-1}=-\gamma$ for $i=2,3, \ldots, n_{1}+n_{2}$. Using these equations, we can calculate section lengths as

$$
\delta_{i}=\frac{1}{\left[i+\frac{r}{1-r}\left(n_{1}+n_{2}\right)\right] \gamma} .
$$

The specific values of $n_{1}$ and $n_{2}$ must be such that the physical parameters of the network are conserved, this is,

$$
\ell=\sum_{i=1}^{n_{1}} \delta_{i}=\sum_{i=1}^{n_{2}} \delta_{n_{1}+i}
$$

By substituting (12) into (13), we obtain

$$
\sum_{i=1}^{n_{1}} \frac{1}{i+\frac{r}{1-r}\left(n_{1}+n_{2}\right)}=\sum_{i=1}^{n_{2}} \frac{1}{i+\frac{1}{1-r}\left(n_{1}+r n_{2}\right)} .
$$

The values of $n_{1}$ and $n_{2}$ that satisfy this equation yield a network partition that is average detectable. Note that as the summands on both side of the equation are different, then it must be $n_{1} \neq n_{2}$.

\section{Problem statement}

In the previous section, we discussed how some simple traffic networks can be given an average detectable representation by dividing each road into several inhomogeneous virtual cells.

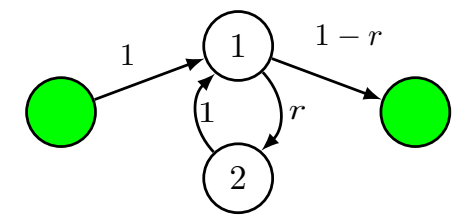

Figure 5: Network representation of a circle road. 


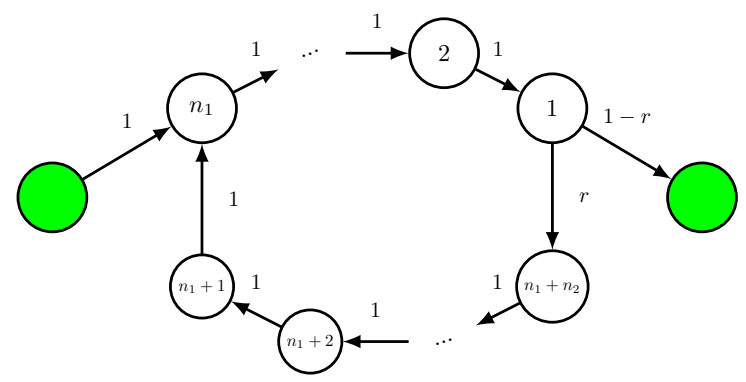

Figure 6: Network of a circle road with virtual partitions. Road 1 is divided into $n_{1}$ sections, and road 2 into $n_{2}$.

For a given traffic network $\mathcal{G}=\{\mathcal{N}, \mathcal{E}, R, \ell, \mathbf{v}\}$ and vector $\mathbf{n} \in \mathbb{N}^{m}$, we introduce the following notation:

Definition: Road division. Consider an arbitrary road $i \in \mathcal{N}$, and $n_{i}$ the corresponding element of $\mathbf{n}$. A division of road $i$ is a directed path graph whose nodes $\left\{i^{(1)}, i^{(2)}, \ldots, i^{\left(n_{i}\right)}\right\}$ are virtual cells of $i$. The downstream cell is denoted $i^{(1)}$, whereas $i^{\left(n_{i}\right)}$ denotes the upstream cell. Additionally, the length and velocity of the $k$-th cell of road $i$ are denoted by $\delta_{i}^{(k)}$ and $v_{i}^{(k)}$, respectively.

Definition: Virtual network. A network $\mathcal{G}^{(\mathbf{n})}=\left\{\mathcal{N}^{(\mathbf{n})}, \mathcal{E}^{(\mathbf{n})}, R^{(\mathbf{n})}, \boldsymbol{\delta}^{(\mathbf{n})}, \mathbf{v}^{(\mathbf{n})}\right\}$ is called a virtual network of $\mathcal{G}$ according to $\mathbf{n}$ if its nodes $\mathcal{N}^{(\mathbf{n})}$ correspond to the road divisions of the nodes $\mathcal{N}$, and the adjacency matrix $R^{(\mathbf{n})}$ satisfies $r_{i^{(1)}, j^{\left(n_{j}\right)}}^{(\mathbf{n})}=r_{i, j}$ and $r_{i^{(j)}, i^{(k)}}^{(\mathbf{n})}=1$ if $k=j-1$. Additionally,

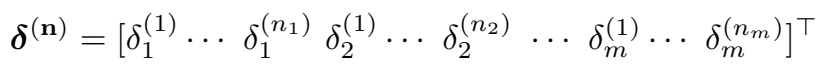

and

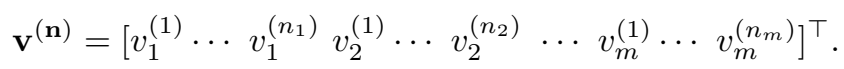

Definition: Admissible virtual network. A virtual network $\mathcal{G}^{(\mathbf{n})}$ of $\mathcal{G}$ is said to be admissible if for every road $i \in \mathcal{N}$, the velocity of any cell is equal to the velocity of the road,

$$
v_{i}^{(k)}=v_{i}
$$

and the sum of cell lengths is equal to the length of the road,

$$
\ell_{i}=\sum_{k=1}^{n_{i}} \delta_{i}^{(k)}
$$

Given a traffic network $\mathcal{G}$, many possible admissible virtual networks $\mathcal{G}^{(\mathbf{n})}$ can be constructed. Each of the cells of the virtual network has an associated density, with its relevant dynamics. Denote as $\rho_{i}^{(k)}$ as the density of the cell $k$ of road $i$, and let $\boldsymbol{\rho}^{(\mathbf{n})}$ be the density vector of dimension $\mathbf{1}^{\top} \mathbf{n}$ corresponding to the virtual network. Thus, the dynamics of the virtual network are given by

$$
\dot{\boldsymbol{\rho}}^{(\mathbf{n})}(t)=A^{(\mathbf{n})} \boldsymbol{\rho}(t)+\operatorname{diag}\left(\boldsymbol{\delta}^{(\mathbf{n})}\right)^{-1} B^{(\mathbf{n})} \mathbf{u}(t)
$$


where

$$
A^{(\mathbf{n})}=\operatorname{diag}\left(\boldsymbol{\delta}^{(\mathbf{n})}\right)^{-1}\left(R^{(\mathbf{n})}-\mathbb{I}\right) \operatorname{diag}\left(\mathbf{v}^{(\mathbf{n})}\right) .
$$

and $B^{(\mathbf{n})}$ maps the input demands to the upstream cell of the boundary inflows.

Following the same discussion as in sections 2.2 and 2.3, we can analyze the convergence of the open-loop observer for the average density of the virtual network, $\rho_{a v}^{(\mathbf{n})}$.

The postulated problem is as follows: for any given traffic network $\mathcal{G}$, find a division vector $\mathbf{n}$, cell length vector $\boldsymbol{\delta}^{(\mathbf{n})}$, and constant $\gamma>0$, such that the virtual network $\mathcal{G}^{(\mathbf{n})}$ is admissible and average detectable.

\section{Virtual division for general networks}

In this section, we present the conditions required for a virtual network to be admissible and average detectable.

Theorem 2. Let $\mathcal{G}=\{\mathcal{N}, \mathcal{E}, R, \boldsymbol{\ell}, \mathbf{v}\}$ be a given traffic network. An admissible network $\mathcal{G}^{(\mathbf{n})}=\left\{\mathcal{N}^{(\mathbf{n})}, \mathcal{E}^{(\mathbf{n})}, R^{(\mathbf{n})}, \boldsymbol{\delta}^{(\mathbf{n})}, \mathbf{v}^{(\mathbf{n})}\right\}$ is average detectable if and only if there exist $\mathbf{n} \in \mathbb{N}^{m}, \gamma>0$, and $\boldsymbol{\delta}^{(\mathbf{n})}$ such that

$$
\delta_{i}^{(k)}=\frac{v_{i}}{\left(v_{i} \mathbf{d}_{i}^{\top} \mathbf{n}+k\right) \gamma}
$$

under the constraints (14) and (15), for all $i=1,2, \ldots, m ; k=1,2, \ldots, n_{i}$; where $\mathbf{d}_{i}^{\top}$ is the $i$-th row of $D=\left(\mathbb{I}-R_{11}\right)^{-1} R_{11} V_{1}^{-1}$.

The proof of Theorem 2 is presented in Appendix A.

Consider a virtual network whose cell lengths are calculated according to (18). Define

$$
f_{i}(\mathbf{n}, \gamma)=\ell_{i}-\frac{v_{i}}{\gamma} \sum_{k=1}^{n_{i}} \frac{1}{v_{i} \mathbf{d}_{i}^{\top} \mathbf{n}+k} .
$$

such that it corresponds to the error in (15), i.e., the error between the sum of cell lengths and the length of road $i$. Thus, the problem of finding an average detectable and admissible division of a given network is equivalent to finding a vector of integers $\mathbf{n}$ and a constant $\gamma$ such that $f_{i}(\mathbf{n}, \gamma)=0$ for all $i=1, \ldots m$. However, this is difficult in practice, as it is a combinatorial problem. As a simplification, we can search for solutions that satisfy the constraints approximatively, that is, to find $\mathbf{n}$ and $\gamma$ such that $\left|f_{i}(\mathbf{n}, \gamma)\right|$ is less than a desired tolerance.

In the following theorems, we propose an alternative system of equations used to calculate $\mathbf{n}$ and $\gamma$. To do this, we allow the values of $\mathbf{n}$ to take real (instead of only integer) values. Then, we approximate the sum in $f_{i}(\mathbf{n}, \gamma)$ using the continuous functions. This results in a system of equations that is simpler to solve, but that results in approximation error. However, we show that this error is bounded and can be reduced by selecting different values of $\gamma$. 
Theorem 3. Consider any given traffic network $\mathcal{G}$. Let $\mathbf{x} \in \mathbb{R}^{m}$ and $\gamma>0$ such that,

$$
\left[\left(K_{\gamma}-\mathbb{I}\right)^{-1} K_{\gamma}-V_{1}\left(\mathbb{I}-R_{11}\right)^{-1} V_{1}^{-1}\right] \mathbf{x}=\frac{1}{2} \mathbf{1},
$$

where $K_{\gamma}=\operatorname{diag}\left(\left[\begin{array}{llll}e^{\gamma \ell_{1} / v_{1}} & e^{\gamma \ell_{2} / v_{2}} & \cdots & e^{\gamma \ell_{m} / v_{m}}\end{array}\right]\right)$. Let $\lfloor\cdot 7$ denote the nearest integer function. Then, $\mathbf{n}=\lfloor\mathbf{x}\rceil$ and $\gamma$ satisfy

$$
\left|f_{i}(\mathbf{n}, \gamma)\right| \sim O\left(\left(v_{i} \mathbf{d}_{i}^{\top} \mathbf{n}+1\right)^{-1}\right)
$$

for $i=1,2, \ldots, m$.

The proof of Theorem 3 is presented in Appendix B.

Theorem 4. There exists $\gamma_{\max }$ such that for every $0<\gamma<\gamma_{\max }$, the solution to (20) is positive. Moreover, as $\gamma$ approaches $\gamma_{\max }$ the magnitude of $\mathbf{x}$ grows arbitrarily large.

The proof of Theorem 4 is presented in Appendix C.

Using Theorems 3 and 4, we propose the following algorithm to find a vector $\mathbf{n}$ of partitions that yields an admissible network within a given tolerance.

Algorithm 1. Create virtual network

Inputs: Traffic network $\mathcal{G}=\{\mathcal{N}, \mathcal{E}, R, \boldsymbol{\ell}, \mathbf{v}\}$. Tolerance $\epsilon$.

Output: Vector of divisions $\mathbf{n}$. Detectability constant $\gamma$.

1. Initialize range for $\gamma$ :

1.1 Set $\gamma_{1} \leftarrow 0$.

1.2 Set $\gamma_{2} \leftarrow \max _{i}\left[-\ln \left(\lambda\left(R_{11}\right)\right) v_{i} / \ell_{i}\right]$.

2. Calculate $\mathbf{n}$ :

2.1 Set $\gamma \leftarrow\left(\gamma_{1}+\gamma_{2}\right) / 2$.

2.2 Set $\mathbf{x} \leftarrow\left[\left(K_{\gamma}-\mathbb{I}\right)^{-1} K_{\gamma}-V_{1}\left(\mathbb{I}-R_{11}\right)^{-1} V_{1}^{-1}\right]^{-1} \mathbf{1} / 2$.

2.3 Set $\mathbf{n} \leftarrow\lfloor\mathbf{x}\rceil$.

2.4 If $\exists i: x_{i}<0$

2.4.1 Set $\gamma_{2} \leftarrow \gamma$.

2.4.2 Go to Step 2.1.

2.5 If $\exists i:\left|f_{i}(\mathbf{n}, \gamma)\right|>\epsilon$

2.5.1 Set $\gamma_{1} \leftarrow \gamma$.

2.5.2 Go to Step 2.1.

\section{Return $\mathbf{n}, \gamma$.}

The algorithm is based on an implementation of the well known bisection method (see Burden and Faires (1985), Ch. 2). From Theorems 3 and 4, as $\gamma$ approaches the maximum value $\gamma_{\max }$, the error function $f_{i}(\mathbf{n}, \gamma)$ decreases its magnitude. As $\gamma_{\max }$ is not generally known a priori, the algorithm searches inside a range of possible values until it finds a value of $\gamma$ for which $f_{i}(\mathbf{n}, \gamma)$ is less than the given tolerance for each $i=1, \ldots, m$. 
The algorithm explores for values of $\gamma$ inside of the interval $\left[\gamma_{1}, \gamma_{2}\right]$, initialized in Step 1. $\gamma_{1}$ is set to zero, which is a trivial constraint from the problem formulation. The expression for the maximum value $\gamma_{2}$ comes from the fact that

$$
\lambda\left(R_{11} K_{\gamma_{\max }}\right)=1
$$

(see proof of Theorem 4), and that $K_{\gamma}$ is a diagonal matrix.

With these initial limits, the algorithm proceeds by setting $\gamma$ as the intermediate value between $\gamma_{1}$ and $\gamma_{2}$, and then it is used to calculate a candidate solution $\mathbf{x}$. If $\mathbf{x}$ has negative entries, it means that the current $\gamma$ is higher than $\gamma_{\max }$, so the upper limit $\gamma_{2}$ is reduced. Else, the error functions $\left|f_{i}(\mathbf{n}, \gamma)\right|$ are compared against the tolerance. If the tolerance is not met, then the lower limit $\gamma_{1}$ is increased. This process continues until a good-enough solution is found.

Note that after every step, the exploration range $\left[\gamma_{1}, \gamma_{2}\right]$ is reduced by half, and therefore, the algorithm will require at most $O\left(\log _{2}(1 / \epsilon)\right)$ cycles. Additionally, every cycle requires the inversion of a $m \times m$ matrix, each requiring $O\left(m^{3}\right)$ calculations. Therefore, the overall computational cost is $O\left(m^{3} \log _{2}(1 / \epsilon)\right)$.

\section{Difference between original and virtual networks}

In the previous sections we have discussed how to generate a virtual network $\mathcal{G}^{(\mathbf{n})}$ from a given one $\mathcal{G}$. The virtual network keeps the same physical properties as the original one, but its roads have been divided into smaller cells. This transformation allows one to have an average detectable network.

Nevertheless, even if the underlying physical network is the same, the dynamics of the real and virtual systems are different, and therefore the trajectories of the corresponding average densities are not identical. Let $\rho_{a v}$ be the average density of the given undivided network, and let $\rho_{a v}^{(\mathbf{n})}$ be the average density of the divided network (following the methods discussed before). It can be seen that both definitions are not equivalent:

$$
\rho_{a v}=\frac{1}{m} \sum_{i=1}^{m} \rho_{i}
$$

where $\rho_{i}$ is the density of road $i$, and,

$$
\rho_{a v}^{(\mathbf{n})}=\frac{1}{\sum_{i=1}^{m} n_{i}} \sum_{i=1}^{m} \sum_{k=1}^{n_{i}} \rho_{i}^{(k)}
$$

where $\rho_{i}^{(k)}$ is the density of cell $k$ of road $i$, and $n_{i}$ is the number of divisions of road $i$. The quantities $\rho_{i}$ and $\rho_{i}^{(k)}$ are related by the expression

$$
\ell_{i} \rho_{i}=\sum_{k=1}^{n_{i}} \delta_{i}^{(k)} \rho_{i}^{(k)}
$$


which states that the total number of vehicles must be the same in both representations.

Figures 7 and 8 show the trajectories of the average density of the real system $\rho_{a v}$, and the virtual system $\rho_{a v}^{(\mathbf{n})}$ for two sample networks considered in this paper.

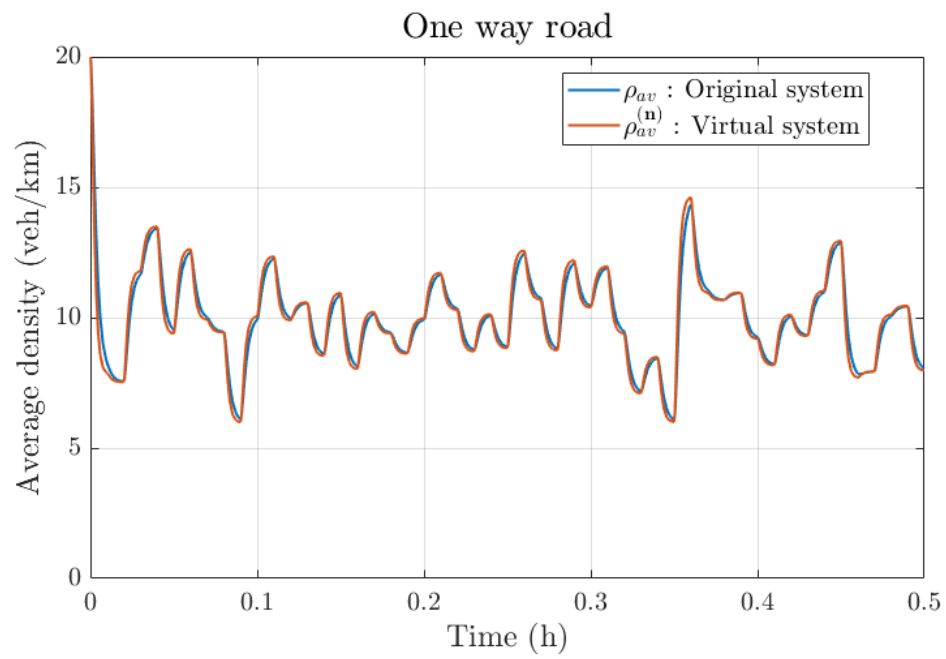

Figure 7: Trajectories of the average densities of the original and virtual systems for the one way road from section 3.1.

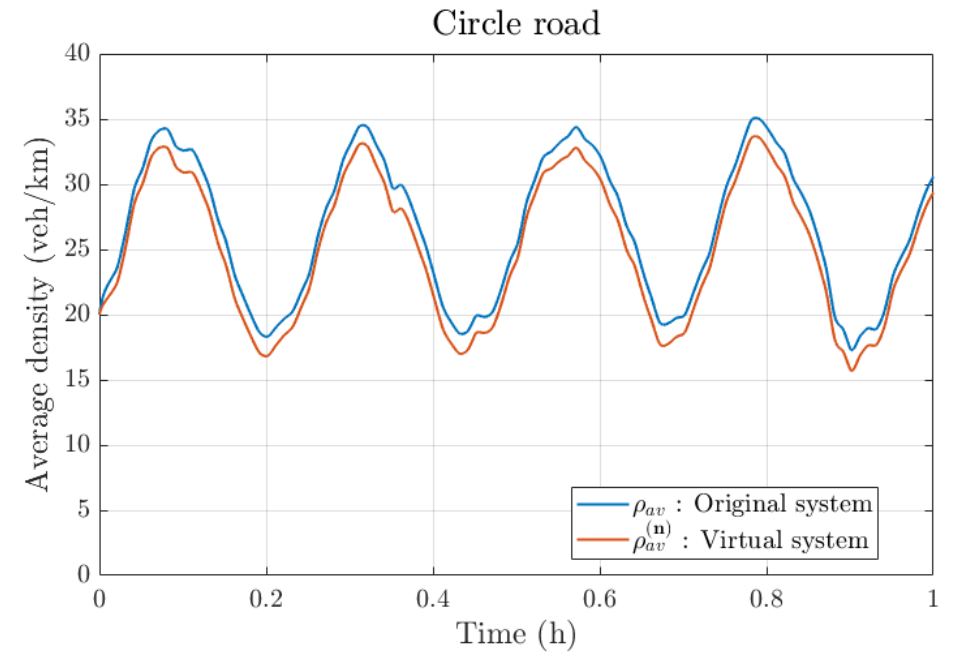

Figure 8: Trajectories of the average densities of the original and virtual systems for the circle road from section 3.2 . 
Note that in general, both trajectories are very close, so using the estimator for the virtual system yields a good result for the real one. However, the closeness between these trajectories might depend in the original network parameters, so it is required to establish a way to determine if a particular network might be well represented using the approach described in this paper.

The error between the average density of the original and virtual system can be found to be

$$
\rho_{a v}^{(\mathbf{n})}-\rho_{a v}=\frac{1}{m} \sum_{i=1}^{m} \sum_{k=1}^{n_{i}}\left(\frac{1}{n_{a v}}-\frac{\delta_{i}^{(k)}}{\ell_{i}}\right) \rho_{i}^{(k)}
$$

where $n_{a v}=\mathbf{1}^{\top} \mathbf{n} / \mathrm{m}$. Note that this expression depends both on the properties of the network division (the cell lengths), and on the state of the virtual network, as the density of each cell is required.

\subsection{Slow varying inputs}

Consider the case where the rate of change of the inputs (incoming flows at the boundaries) is small in comparison to the traveling time of each road. This can be achieved if the input demands are relatively constant, or in urban scenarios where the distance between intersections is small. Note that this does not imply that the physical dimensions of the entire network is small, just its individual components. This assumption implies that there are small spatial variations in the flow for each road, so that $\varphi_{\text {in }}(t) \approx \varphi_{\text {out }}(t)$. This in turn implies that the density of each road is approximately constant in space, and thus

$$
\rho_{i}^{(k)} \approx \rho_{i}
$$

for each road $i$ and each cell $k$. It follows that the average density of the virtual system can be approximated as a weighted average of the density of the internal nodes, where the weights are the number of partitions,

$$
\rho_{a v}^{(\mathbf{n})} \approx \frac{1}{n_{t o t}} \mathbf{n}^{\top} \boldsymbol{\rho}_{1} .
$$

where $n_{t o t}=\mathbf{1}^{\top} \mathbf{n}$. Thus, we can simplify the error equation as

$$
\rho_{a v}^{(\mathbf{n})}-\rho_{a v} \approx \frac{1}{m} \sum_{i=1}^{m} \rho_{i}\left(\frac{n_{i}}{n_{a v}}-1\right) .
$$

Note that in this case, the difference between the two trajectories depends only on the densities of the roads (not cells), and the number of cells per road. Using matrix notation, this can be rewritten as

$$
\rho_{a v}^{(\mathbf{n})}-\rho_{a v} \approx \frac{\mathbf{n}^{\top} \boldsymbol{\sigma}}{\mathbf{n}^{\top} \mathbf{1}}
$$

where

$$
\boldsymbol{\sigma}=\boldsymbol{\rho}-\bar{\rho}_{a v} \mathbf{1} .
$$


The value of the error depends then on the cell division and the state of the original system, which is unknown. To benchmark the applicability of the proposed method for all possible networks, we can analyze the distribution of the error under the assumption that the deviation $\boldsymbol{\sigma}$ is a random process. We introduce the following theorem,

Theorem 5. Consider the metric space $\left(\mathbb{S}^{m}, d\right)$ where $\mathbb{S}^{m}=\left\{\mathbf{x} \in \mathbb{R}^{m}:\|\mathbf{x}\|=\right.$ $1\}$ is the $m$-dimensional unit sphere and $d$ is the spherical distance. Consider two independent random vectors $\mathbf{u} \in \mathbb{S}^{m}$ and $\mathbf{v} \in \mathbb{S}^{m}$. Then, for any $\epsilon>0$,

$$
P_{\mathbf{u}, \mathbf{v}}\left(\left|\mathbf{u}^{\top} \mathbf{v}\right|<\epsilon\right) \geq 1-2 e^{-(m-1) \epsilon^{2} / 2}
$$

where $P$ is the uniform spherical probability measure.

The proof of Theorem 5 is shown in Appendix D. This implies that for large dimensions $(m \rightarrow \infty)$, the probability of the two vectors being $\epsilon$-orthogonal is asymptotically 1 .

Because of this, the trajectories of the average density of the original and virtual systems get closer as we consider larger networks. Note that this depends on the assumption $\boldsymbol{\sigma}$ is independent to $\mathbf{n}$. Even though both vectors depend on the properties of the network, this assumption can be justified by the fact that $\boldsymbol{\sigma}$ depends highly on the input demands $\mathbf{u}$, and thus, the orientation of $\boldsymbol{\sigma}$ does not depend on the orientation of $\mathbf{n}$.

\section{Application scenario 1: Manhattan grid network}

Consider the traffic network shown in Fig. 9, which corresponds to the network for a Manhattan Grid of $4 \times 4$ intersections. Assume that all roads have the same length of $\ell=500 \mathrm{~m}$, and the same free-flow velocity of $v=30 \mathrm{~km} \cdot \mathrm{h}^{-1}$. As all speeds and lengths are equal, $K_{\gamma}=\exp \left(\gamma \frac{\ell}{v}\right) \mathbb{I}$, and $\gamma_{\max }=-(v / \ell) \ln \left[\lambda\left(R_{11}\right)\right]$. 


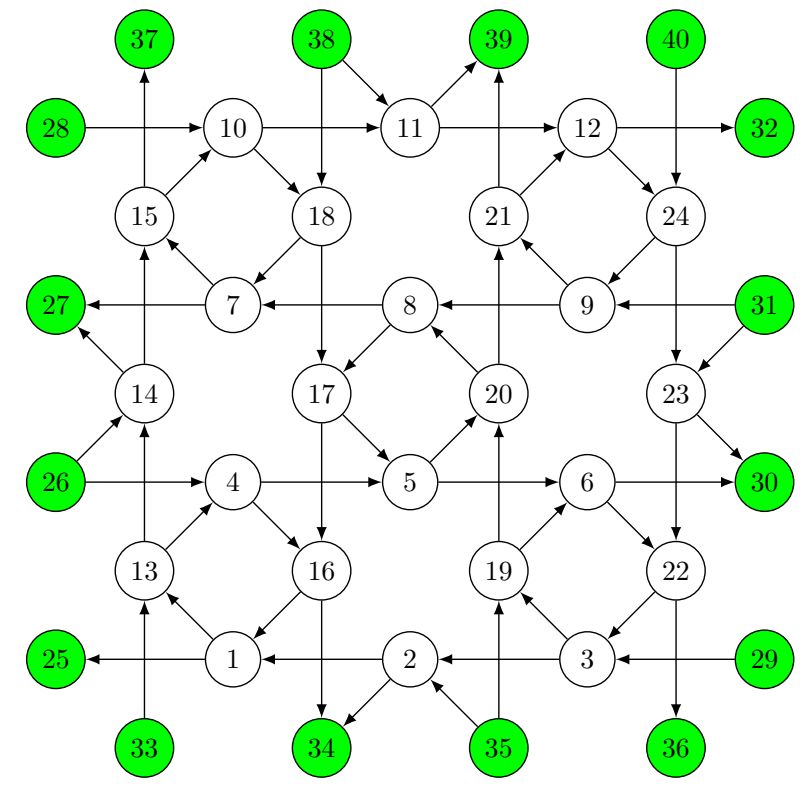

Figure 9: $4 \times 4$ Manhattan grid (nodes correspond to roads). All turning ratios are set to $50 \%$. Green nodes symbolize sensor locations.

It was shown in the previous section that for a given value of $\gamma \in\left(0, \gamma_{\max }\right)$, there exists one vector $\mathbf{n}$ that is an approximate solution to the problem. Figure 10 shows $\mathbf{n}$ that solves (20) for different values of $\gamma$. For $\gamma<0.88 \gamma_{\max }$, the elements of $\mathbf{n}$ are all 1. As $\gamma$ approaches the maximum value, $\mathbf{n}$ increases quickly.

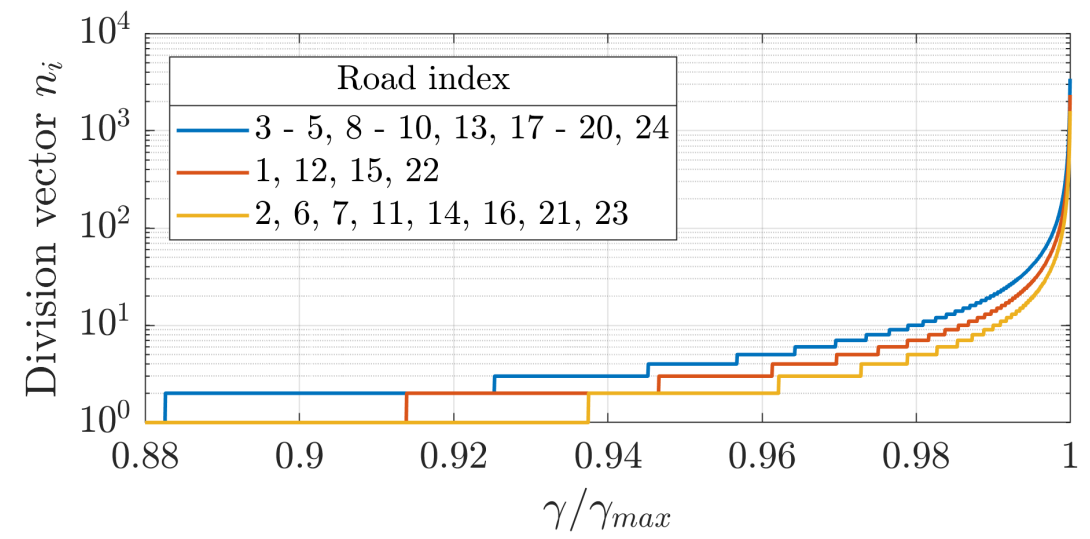

Figure 10: Approximate solutions of (20) for $\mathbf{n}$ for all unmeasured nodes.

However, using (20) to calculate $\mathbf{n}$ induces an error $f_{i}(\mathbf{n}, \gamma)$ in the admissibility constraint. Consider the total root mean square error (RMSE) for all 
roads $i=1, \ldots, m$, as shown in Fig. 11. As $\gamma$ increases, the upper limit of this error decreases, approaching 0 as $\gamma \rightarrow \gamma_{\max }$. This is because the number of cells per road is also increasing rapidly, so Theorem 3 is applicable. In this sense, the lowest error is obtained by choosing $\gamma$ very close to $\gamma_{\max }$.

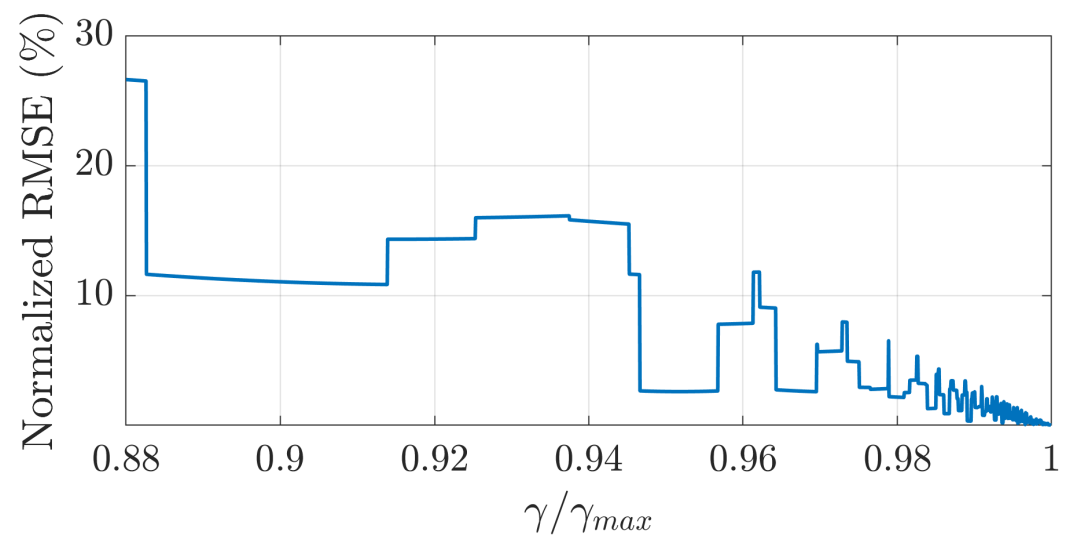

Figure 11: Normalized RMSE, $\sqrt{\sum_{i} f_{i}(\mathbf{n}, \gamma)^{2}} / \ell$ for different values of $\gamma$.

Note that the virtual networks that yield a low error in the admissibility constraint require many divisions per road and therefore are high-dimensional. Using the defined observer in sections 2.2 and 2.3 applied to the dynamics of the virtual system in (16), we obtain an estimator for the average density of the virtual system of the form

$$
\dot{\hat{\rho}}_{a v}^{(\mathbf{n})}(t)=\frac{1}{n_{t o t}} \mathbf{1}^{\top} A_{11}^{(\mathbf{n})} \mathbf{1} \hat{\rho}_{a v}^{(\mathbf{n})}+\frac{1}{n_{t o t}} \mathbf{1}^{\top} A_{12}^{(\mathbf{n})} \mathbf{y}(t)
$$

where $n_{t o t}=\mathbf{1}^{\top} \mathbf{n}$. The dimensions of the matrices $A_{11}^{(\mathbf{n})}$ and $A_{12}^{(\mathbf{n})}$ are proportional to the norm of $\mathbf{n}$, hence, large number of divisions could imply costly calculations to obtain the values required by the estimator. However, the expressions for the gains in the estimator can be simplified such that the matrix $A^{(\mathbf{n})}$ is not explicitly required.

The first term can be simplified by using the fact that the network is average detectable, which requires $\mathbf{1}^{\top} A_{11}^{(\mathbf{n})}=-\gamma \mathbf{1}^{\top}$ and thus

$$
\frac{1}{n_{t o t}} \mathbf{1}^{\top} A_{11}^{(\mathbf{n})} \mathbf{1}=-\frac{\gamma}{n_{t o t}} \mathbf{1}^{\top} \mathbf{1}=-\gamma
$$

The second term of the estimator can also be simplified. Let $\mathbf{b}^{\top}=\frac{1}{n_{\text {tot }}} \mathbf{1}^{\top} A_{12}^{(\mathbf{n})}$ be the vector gain for the measurements. From the definition of this matrix, we obtain:

$$
b_{i-n_{\text {tot }}}=\frac{1}{n_{t o t}} \sum_{j=1}^{m} \sum_{k=1}^{n_{j}} r_{i, j^{(k)}}^{(\mathbf{n})} \frac{v_{i}}{\delta_{j}^{(k)}}
$$


where the indexes $i=n_{t o t}+1, \ldots, n_{t o t}+k$ correspond to the measured boundary nodes in the virtual network. From the definition of $R^{(\mathbf{n})}$, the expression can be simplified to

$$
b_{i-n_{t o t}}=\frac{1}{n_{t o t}} \sum_{j=1}^{m} r_{i, j} \frac{v_{i}}{\delta_{j}^{\left(n_{j}\right)}} .
$$

Substituting (18) into (35),

$$
b_{i-n_{\text {tot }}}=\frac{\gamma v_{i}}{n_{t o t}} \sum_{j=1}^{m} r_{i, j}\left(\mathbf{d}_{j}^{\top} \mathbf{n}+\frac{n_{j}}{v_{j}}\right) .
$$

Using matrix notation, this can be rewritten into

$$
\mathbf{b}^{\top}=\frac{\gamma}{\mathbf{1}^{\top} \mathbf{n}} \mathbf{n}^{\top}\left(D^{\top}+V_{1}^{-1}\right) R_{21}^{\top} V_{2}
$$

Substituting the definition of $D$, this simplifies to

$$
\mathbf{b}^{\top}=\frac{\gamma}{\mathbf{1}^{\top} \mathbf{n}} \mathbf{n}^{\top} V_{1}^{-1}\left(\mathbb{I}-R_{11}^{\top}\right)^{-1} R_{21}^{\top} V_{2} .
$$

Hence, the estimator for the average density of the virtual system becomes

$$
\dot{\hat{\rho}}_{a v}^{(\mathbf{n})}(t)=-\gamma \hat{\rho}_{a v}^{(\mathbf{n})}(t)+\frac{\gamma}{\mathbf{n}^{\top} \mathbf{1}} \mathbf{n}^{\top} V_{1}^{-1}\left(\mathbb{I}-R_{11}^{\top}\right)^{-1} R_{21}^{\top} V_{2} \mathbf{y}(t) .
$$

Note that to use the proposed observer, only the calculation of $\mathbf{n}, \gamma$, and the topology of the original network is required. As the virtual network is not required, this provides an efficient way to deploy the estimator even for very high number of divisions. Figure 12 shows the values of $\mathbf{b}^{\top}$ as a function of $\gamma$.

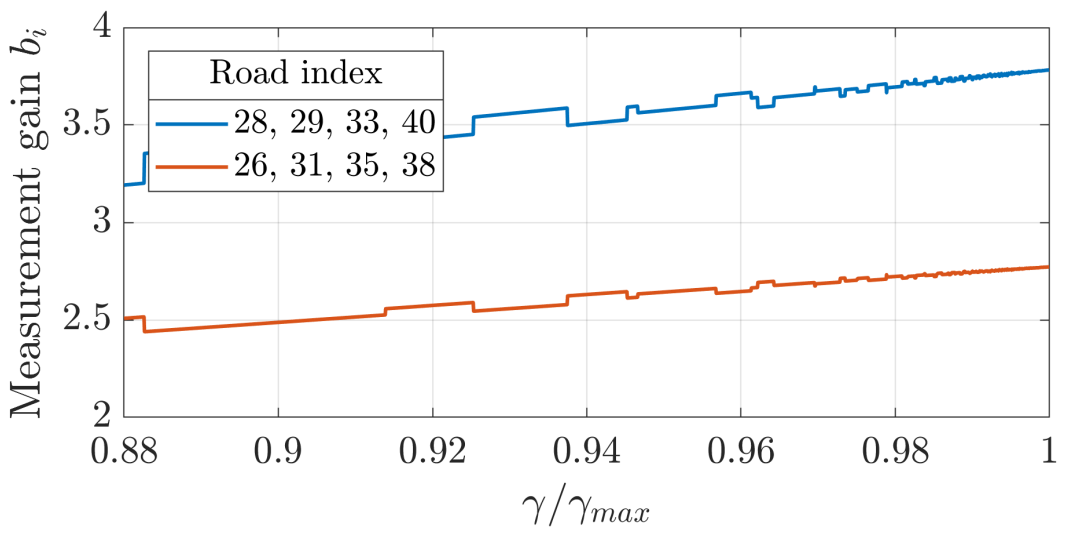

Figure 12: Measurement gain for the open-loop observer as a function of $\gamma$.

As a specific case, let $\gamma=0.95 \gamma_{\max }$, which corresponds to a vector $\mathbf{n}$ with elements 2, 3 and 4, and a RMS error below $3 \%$. The corresponding virtual 


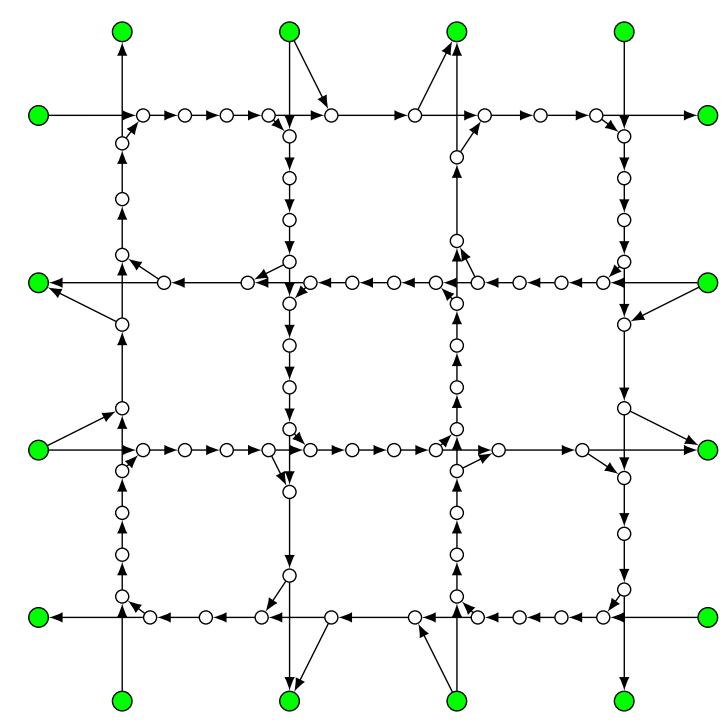

Figure 13: Virtual network using $\gamma=0.95 \gamma_{\max }$.

network is shown in Fig. 13. Using the virtual network as an input, we performed a simulation using random initial conditions, and sinusoidal inputs with additive noise. The trajectory of the real average density is shown in blue in Fig. 14.

Using the measurements $\mathbf{y}$ as an input, we used the open-loop observer to estimate the average density. The trajectory of the estimate is shown in red in Fig. 14. This observer converges to the real solution as expected. Note that to deploy the observer, the virtual network of Fig. 13 is not needed; only $\gamma, \mathbf{n}$ are required and can be calculated off-line. Therefore, the on-line deployment of the observer requires little computational power and is applicable for large-scale networks. 


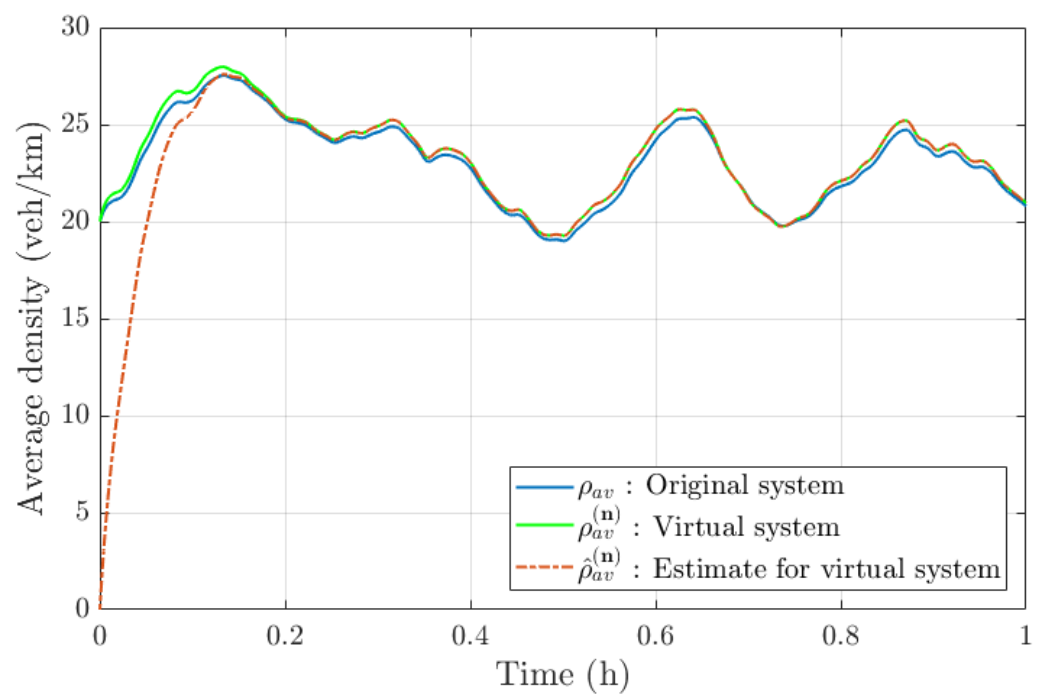

Figure 14: Real and estimated average density.

\section{Application scenario 2: Grenoble Downtown}

This work makes part of the Scale-FreeBack ${ }^{1}$ research project, which deals with the modeling, monitoring and control of large scale systems. One of its initiatives oriented to the application to traffic networks is the GTL-Ville ${ }^{2}$, which takes as subject the city of Grenoble. The GTL-Ville serves as a platform to share data and analysis with the users of the traffic network, and to constitute a collection of validation experiments for the development of traffic research. All data and analysis are available for download. Figure 15 shows the home website of the GTL-Ville. From here, users can inspect available data from sensors and traffic indicators for roads.

For the purposes of this paper, we consider a section of the Grenoble network with dimensions $1.4 \mathrm{~km}$ by $1 \mathrm{~km}$, as shown in Fig. 16. This area is equipped with two type of sensing technologies:

- magnetic loops, located under the pavement, detect the presence of vehicles by measuring a change in inductance, and can provide the number of vehicles that passed the location during a time interval.

- radar sensors, which emit a pulse of infrared light and then collect the reflected light. By measuring the time between pulses, these sensors can provided the velocity and length of individual vehicles, and the vehicular flow in the location.

\footnotetext{
${ }^{1}$ http://scale-freeback.eu/

${ }^{2}$ http://gtlville.inrialpes.fr/
} 


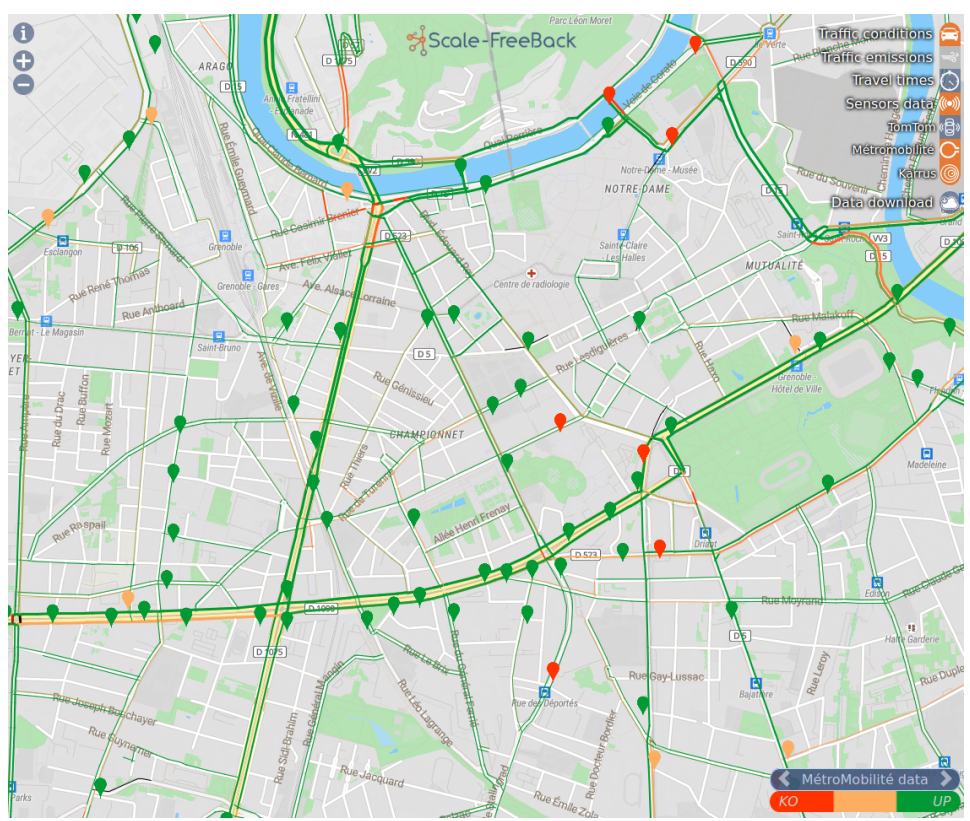

Figure 15: Home website of the GTL-Ville.

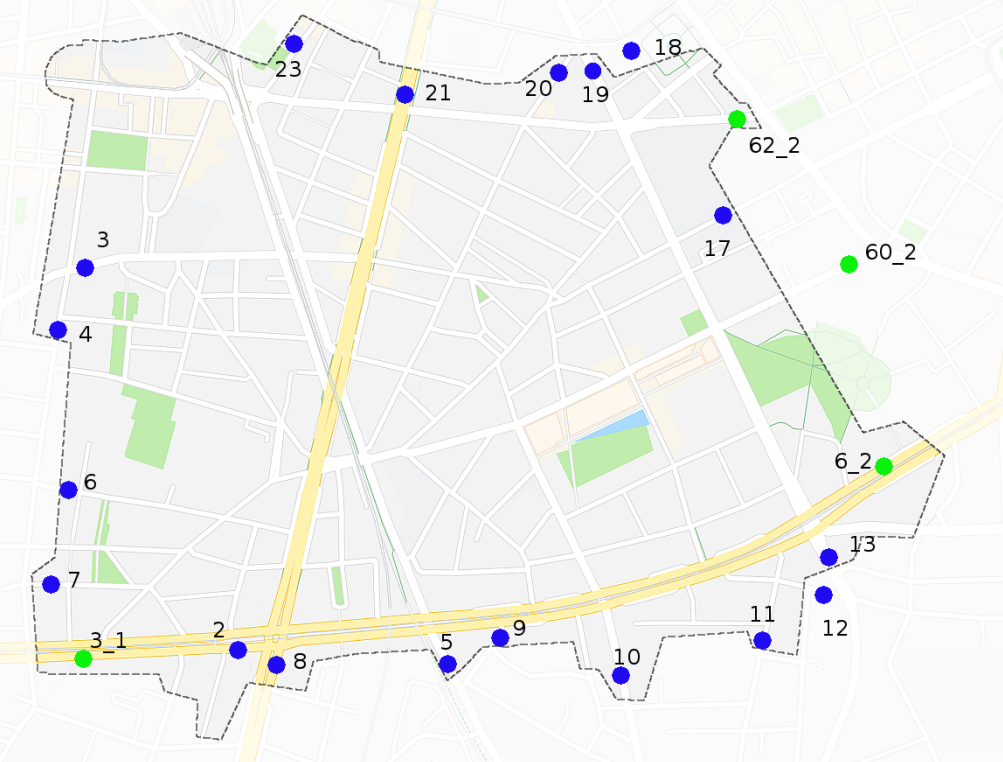

Figure 16: Selected area of the Grenoble traffic network. Blue: radar locations, Green: magnetic loop locations. Text indicates sensors identifier in database. 


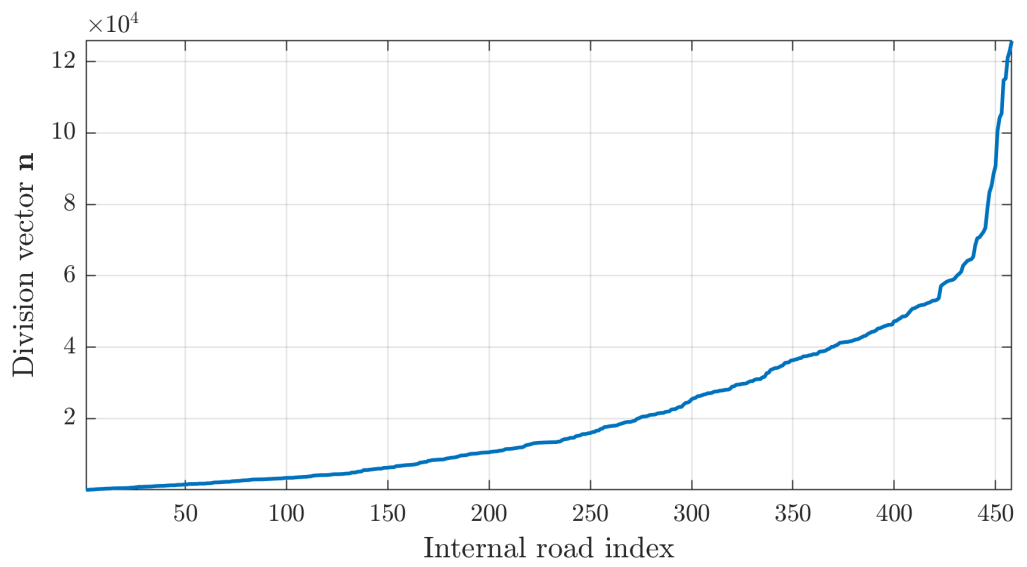

Figure 17: List of values of the division vector $\mathbf{n}$ sorted in ascending order.

The figure shows the location of the sensors that will be used as an input to the proposed method. These locations correspond to most of the boundary roads of the area.

In addition to the sensor data, aggregated FCD provides the average speed data for each road. This data is provided by TomTom ${ }^{3}$, a navigation assistance company.

Because of the network's size it is not feasible to analyze the variation of the division vector $\mathbf{n}$ and error functions $\mathbf{f}(\gamma, \mathbf{n})$ for all possible values of $\gamma$. To calculate the number of partitions per road, we used Algorithm 1, with an error tolerance of $5 \%$. Figures 17 and 18 shows the resulting division vector. For this set up, the algorithm required 24 iterations to satisfy the constraints.

Note that this result implies that roads are divided into very wildly varying number of cells, for which the maximum requires around 12000 cells. Nevertheless, as shown in the previous simulated example, the deployment of the estimator only requires the outputs of the algorithm $(\mathbf{n}$ and $\gamma)$ and the properties of the original network. Therefore, the very large number of cells in the virtual representation does not lead to a problem in the actual on-line calculations.

Although sensors are available at the network's boundaries, neither the density of each road nor the average network's density are known. For this reason, to be able to validate the methods it is required to use simulated data. To obtain a more realistic scenario, we used the well-known microscopic simulator Aimsun, Barceló and Casas (2005). This type of simulators models the behavior of each individual vehicle, depending on its relative position and velocity with respect to other surrounding vehicles. Therefore, this allows to obtain behavior not easily captured by macroscopic models, such as vehicle slowing down at intersections, stop and go waves, and other phenomena.

\footnotetext{
${ }^{3}$ https://www.tomtom.com
} 


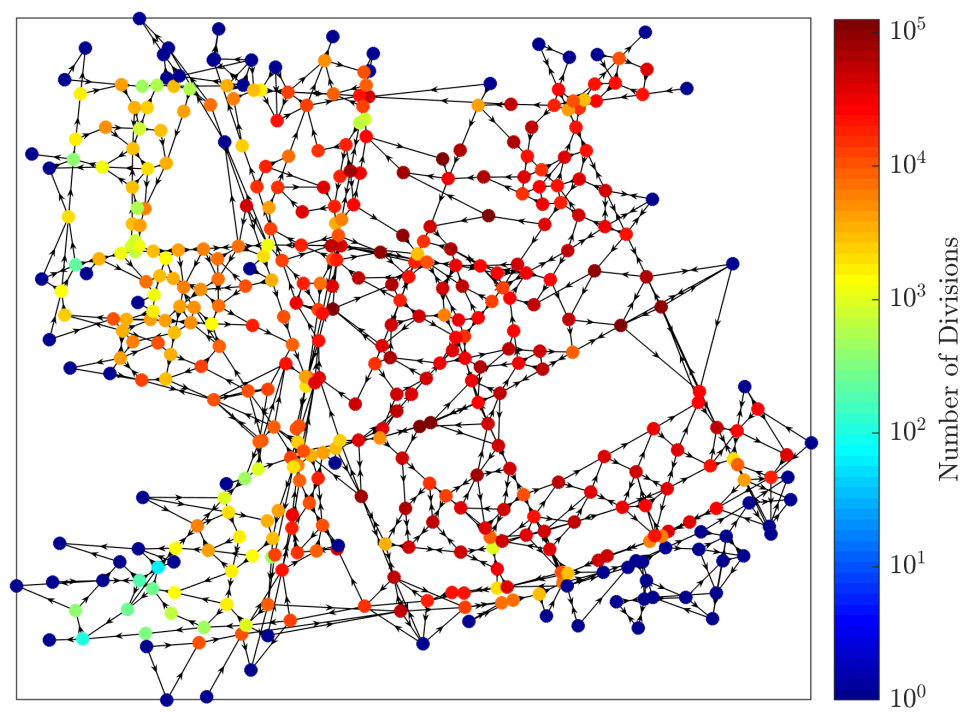

Figure 18: Network representation of the selected area where the color of each node corresponds to its number of cells. The color scale of this graph is logarithmic.

To use Aimsun, we recreated the layout of the Grenoble network as shown in Fig. 19. Sensor data is used to feed the simulator with a real inflow profile. To determine the turning ratios, we use the heuristic

$$
r_{i, j}=\frac{v_{j} \Gamma_{j}}{\sum_{k \in \mathcal{O}(i)} v_{k} \Gamma_{k}}
$$

where $\Gamma_{i}$ is the number of lanes of road $i$, and $\mathcal{O}(i)$ is the set of downstream roads adjacent to $i$. This heuristic is based on the assumption that turning ratios are proportional to the maximum capacity of the receiving road.

The simulation outputs are: density, mean speed, inflow and outflow for every road. They are aggregated for $5 \mathrm{~min}$. From this data, we compute the real average density for the internal (non-boundary) roads as

$$
\rho_{a v}=\frac{1}{m} \mathbf{1}^{\top} \boldsymbol{\rho}_{1}[k] .
$$

As specified in Section 2.1.1, the proposed estimator was designed using a linear model, assuming the velocity of each road being constant. However, in common urban traffic networks applications, this is not the case. Therefore, it is necessary to evaluate the resulting errors caused by the deviation of this hypothesis.

Consider

$$
\rho_{i}^{*}=\max _{k}\left(\rho_{i}[k]\right)
$$




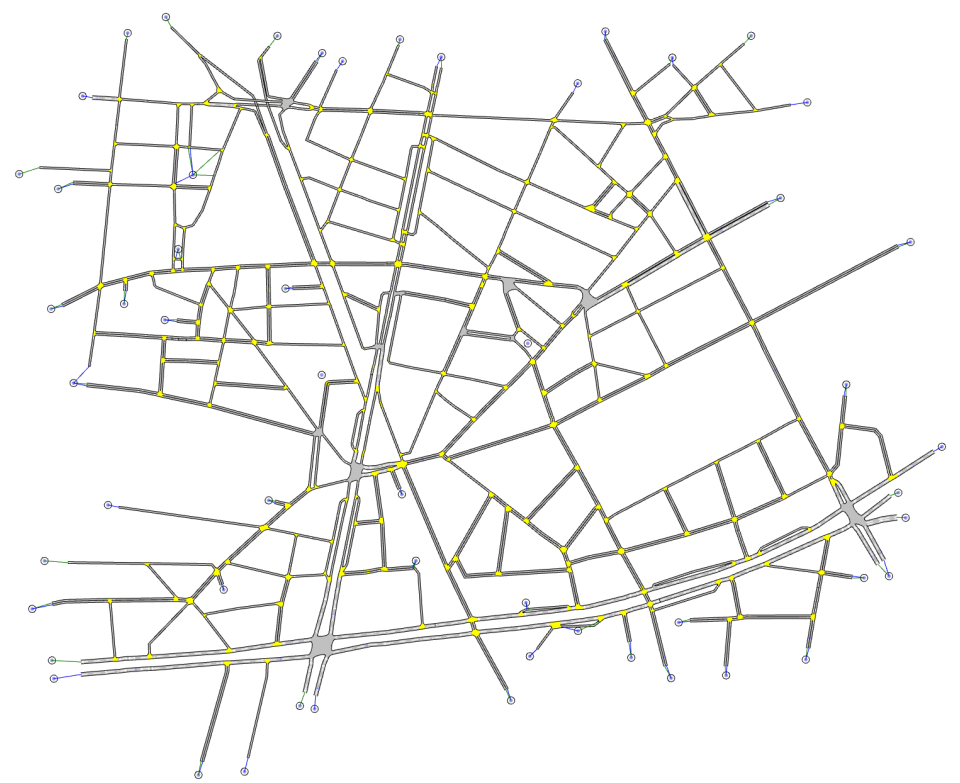

Figure 19: Recreated layout of the Grenoble network in Aimsun. For each road, parameters include the number of lanes, speed limit, length, and road interconnections.

as the maximum density obtained in road $i$. Figure 20 shows the spatial distribution of the $\boldsymbol{\rho}^{*}$ for a simulation. Note that several locations present congestion (the density is higher than the critical value), specially at the center of the network. As a result, the average vehicle speed of the congested roads varies in time. For instance, Fig. 21 shows the speed profile of the road with the largest density $\rho_{i}^{*}$. The deviation from the speed limit is significant, passing from 35 $\mathrm{km} / \mathrm{h}$ to a minimum of about $7 \mathrm{~km} / \mathrm{h}$.

Figure 22 shows the trajectories of the real average density and the estimated value for the virtual system.

Consider the estimation error calculated by

$$
\frac{\sum_{k}\left|\rho_{a v}[k]-\rho_{a v}^{(\mathbf{n})}[k]\right|}{\sum_{k} \rho_{a v}[k]} .
$$

For the current scenario, we obtained an error of $10.2 \%$. This error is due to the speed deviations from the speed limit. This can be seen as between $6 \mathrm{~h} 00$ and $8 \mathrm{~h} 00$, the estimation error is low, and then increases after $8 \mathrm{~h} 00$, which corresponds to the same time when the speed in Fig. 21 deviates from its nominal value.

To verify this results, a second simulation was performed, where the input demands were increased. Figure 23 shows the spatial distribution of the density 


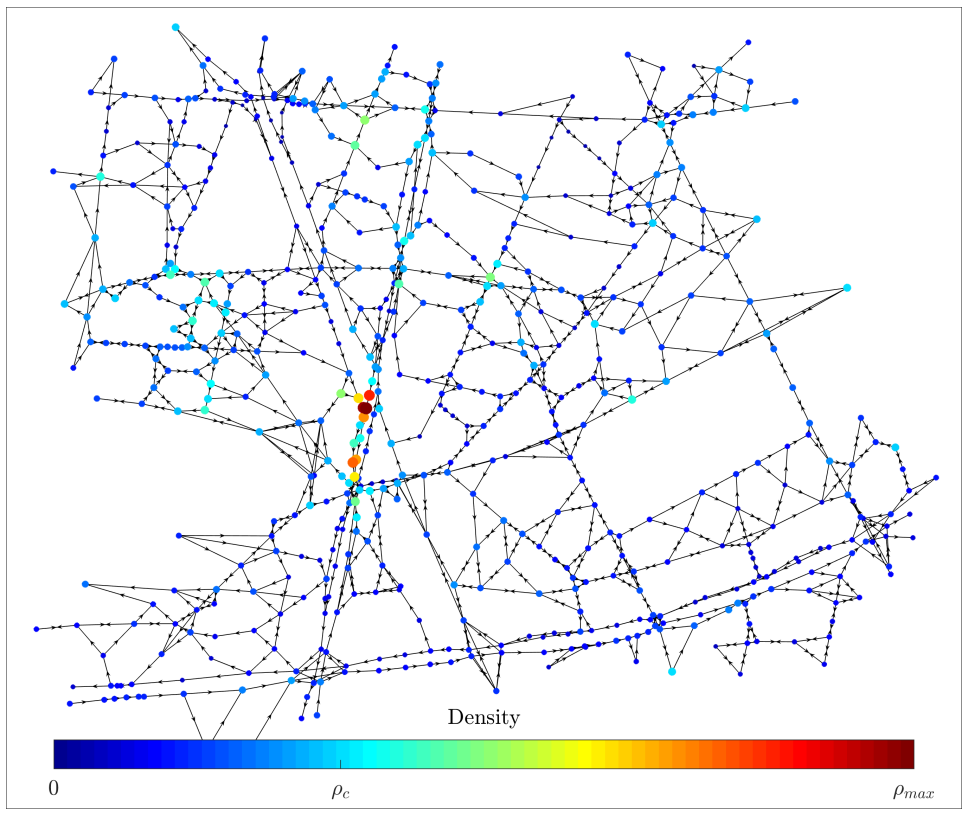

Figure 20: Spatial distribution of the highest densities for simulation 1.

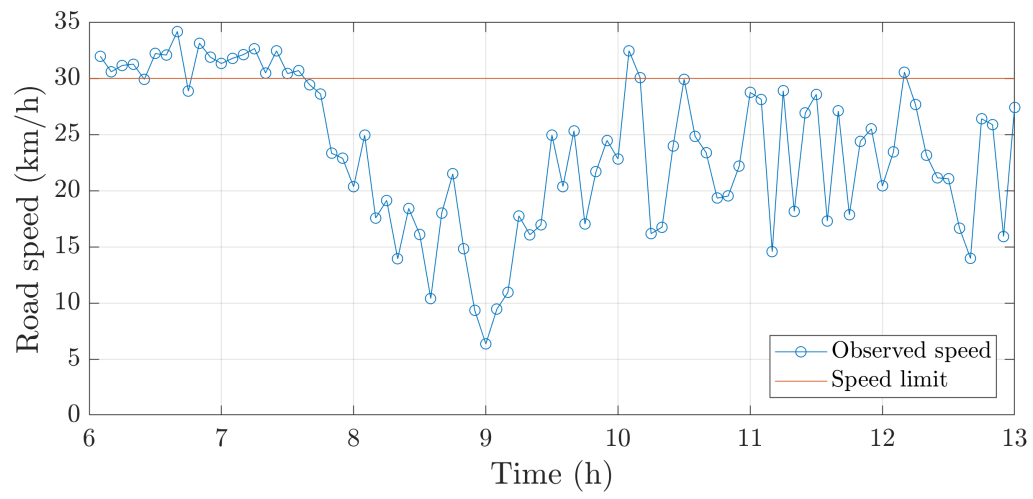

Figure 21: Average vehicle velocity for a selected road for simulation 1. 


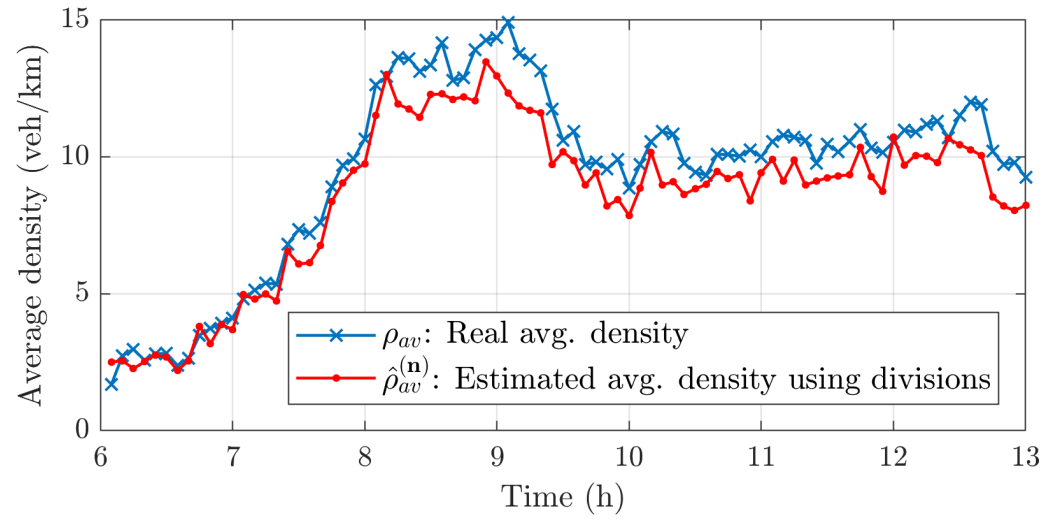

Figure 22: Average density trajectories for simulation 1.

values. Note that the presence of new congested areas specially at the north and north-east. Similarly, the speed profile in Fig. 24 shows a significant decrease in the average vehicle velocities during the congestion, which in turns results in an error in the estimation of the average density, as seen in Fig. 25. The relative error obtained for this simulation is now of $8.8 \%$.

To generalize the relation between the estimation error and the speed deviation, denote by $V_{1}^{*}$ the real speed of internal roads, and $V_{1}$ the nominal speed used in the estimator. By assuming the steady-state of (39), the estimation error is

$$
\rho_{a v}^{(\mathbf{n})}-\hat{\rho}_{a v}^{(\mathbf{n})} \approx \frac{1}{n_{t o t}} \mathbf{n}^{\top}\left(\bar{V}_{1}^{-1}-V_{1}^{-1}\right) \mathbf{w}
$$

where $\mathbf{w}=\left(\mathbb{I}-R_{11}^{\top}\right)^{-1} R_{21}^{\top} V_{2} \mathbf{y}$. This equation provides just an approximation of the estimation error, as it assumes steady-state conditions. However, this allows to identify key factors in the determination of the error.

\section{Conclusion}

In this work, we propose a novel approach to modify a given urban traffic network by dividing each road into cells, such that there exists a one-dimensional open-loop observer that estimates the modified traffic networks's average density. Exact conditions relating the number of cells per road and their lengths are provided. However, these conditions are difficult to be satisfied exactly in some practical cases. To solve this situation, we also propose a procedure to find approximate solutions. As we decrease the approximation error, the number of required partitions increases quickly, therefore implying a virtual network of large dimension. However, to deploy the open-loop observer requires few parameters which can be obtained without explicitly calculating the entire virtual system. 


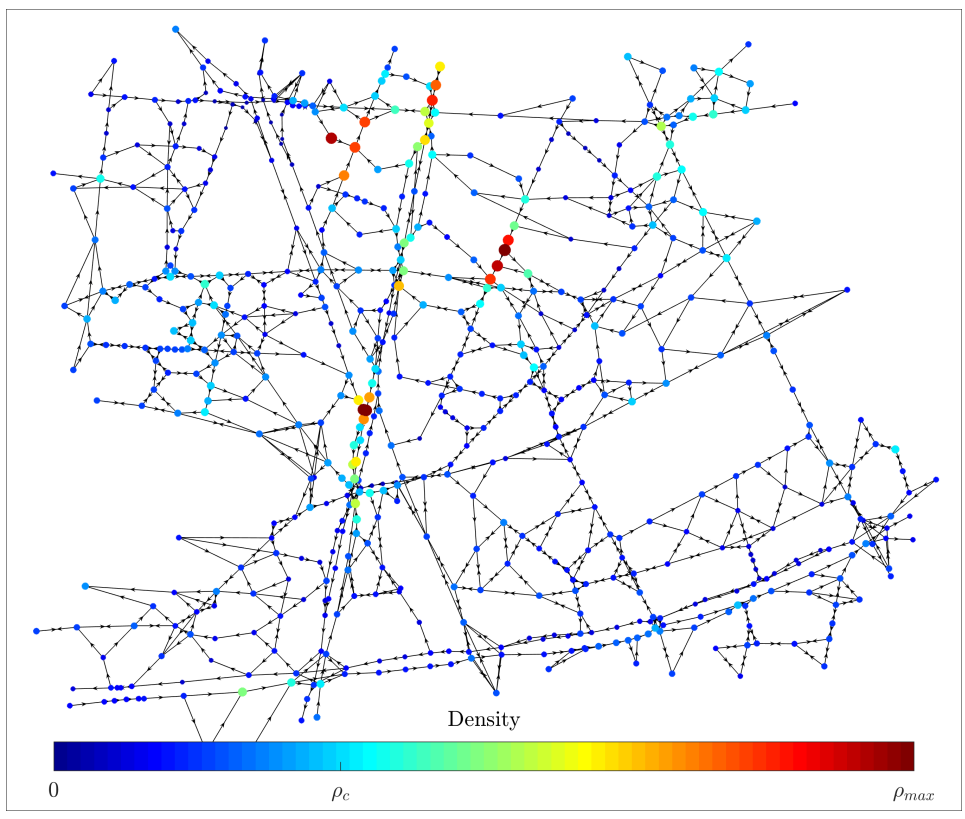

Figure 23: Spatial distribution of the highest densities for simulation 2.

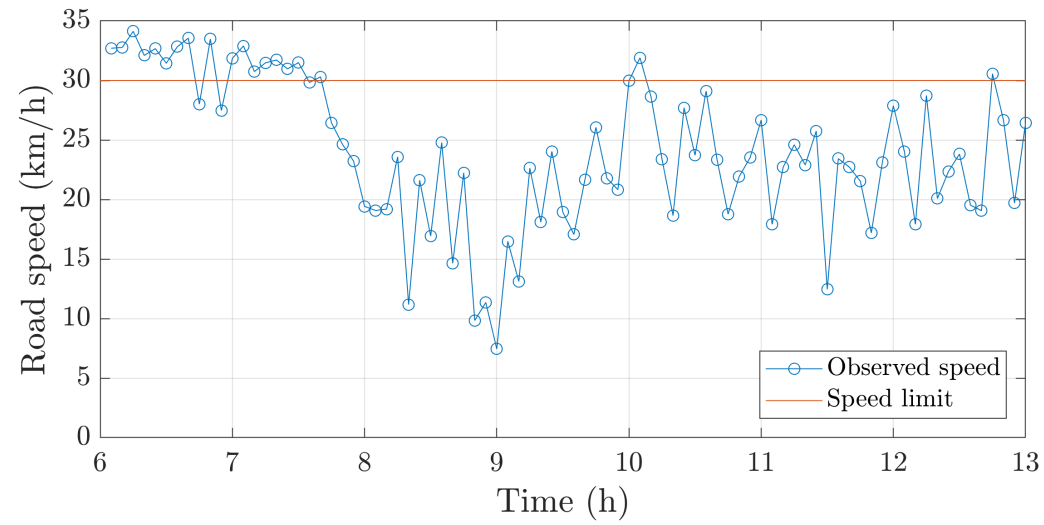

Figure 24: Average vehicle velocity for a selected road for simulation 2. 


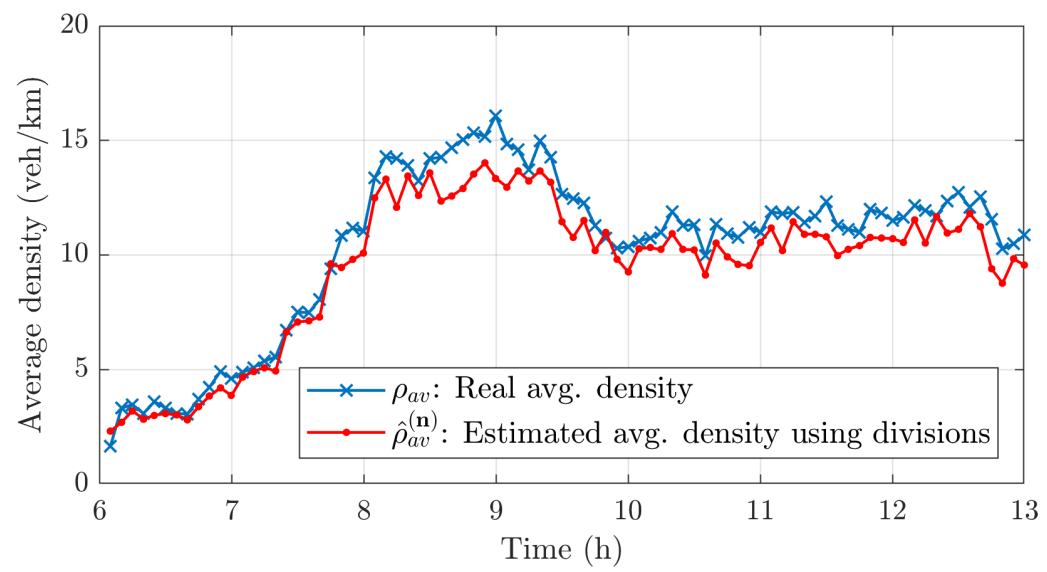

Figure 25: Average density trajectories for simulation 2.

We also analyzed the difference between the average densities of the real system (for which the observer does not generally converge) and the virtual system. The difference between the two trajectories depends not only on the number of cells and their length, but also on the density of every individual road, which are unknown. Nevertheless, we show that by considering large networks, the difference between these two variables is almost always small, and thus, the method can give a useful approximation to the value of interest.

We showed the applicability of the results and the algorithm using two simulated networks. The first considers a Manhattan grid in free-flow, and the second the real case of a zone of the city of Grenoble in France.

For future works, we will analyze how to generalize the average-density estimator to account for the presence of full congestion. This can be done with the aid of measurements of the average vehicle speed in each road from FCD.

\section{Acknowledgements}

This project has received funding from the European Research Council (ERC) under the European Union's Horizon 2020 research and innovation programme (grant agreement 694209), http://scale-freeback.eu.

\section{Appendix A. Proof of Theorem 2}

Consider an arbitrary cell $i^{(k)}$, with $k \neq 1$ such that its downstream neighbor is cell $i^{(k-1)}$. The column sum of $A_{11}^{(\mathbf{n})}$ corresponding to this cell is

$$
-\frac{v_{i}}{\delta_{i}^{(k)}}+\frac{v_{i}}{\delta_{i}^{(k-1)}}=-\gamma .
$$


where we imposed the condition $v_{i}^{(k)}=v_{i}$. By induction, we can calculate the length of each cell from $\delta_{i}^{(1)}$,

$$
\frac{1}{\delta_{i}^{(k)}}=\frac{1}{\delta_{i}^{(1)}}+\frac{k-1}{v_{i}} \gamma
$$

Cell $i^{(1)}$ has as out-neighbors all cells $j^{\left(n_{j}\right)}$ such that $(i, j) \in \mathcal{E}$. Thus, its corresponding column sum is,

$$
-\frac{v_{i}}{\delta_{i}^{(1)}}+\sum_{j=1}^{m} \frac{r_{i j} v_{i}}{\delta_{j}^{\left(n_{j}\right)}}=-\gamma .
$$

Define $\boldsymbol{\delta}_{(1)}^{-1}=\left[\begin{array}{llll}1 / \delta_{1}^{(1)} & 1 / \delta_{2}^{(1)} & \cdots & 1 / \delta_{m}^{(1)}\end{array}\right]$. By substituting (A.1) into (A.2), we obtain a system of linear equations,

$$
\left(\mathbb{I}-R_{11}\right) \boldsymbol{\delta}_{(1)}^{-1}=\gamma\left[R_{11} V_{1}^{-1} \mathbf{n}+\left(\mathbb{I}-R_{11}\right) V_{1}^{-1} \mathbf{1}\right] .
$$

Thus,

$$
\frac{1}{\delta_{i}^{(1)}}=\gamma\left(\mathbf{d}_{i}^{\top} \mathbf{n}+\frac{1}{v_{i}}\right)
$$

are the solutions to (A.3) for the downstream cells of each road $i$. Substitution of (A.4) into (A.1) gives (18).

\section{Appendix B. Proof of Theorem 3}

Let $\psi$ be the digamma function. Its definition and a list of properties can be found in Abramowitz and Stegun (1972). This function satisfies the following identity,

$$
\sum_{k=1}^{n} \frac{1}{z+k}=\psi(z+n+1)-\psi(z+1) .
$$

Therefore, with $z=v_{i} \mathbf{d}_{i}^{\top} \mathbf{n}$, (19) can be rewritten as

$$
f_{i}(\mathbf{n}, \gamma)=\ell_{i}-\frac{v_{i}}{\gamma}\left[\psi\left(v_{i} \mathbf{d}_{i}^{\top} \mathbf{n}+n_{i}+1\right)-\psi\left(v_{i} \mathbf{d}_{i}^{\top} \mathbf{n}+1\right)\right] .
$$

Define $\epsilon(z)=\psi(z)-\ln \left(z-\frac{1}{2}\right)$. It is known that for $z>\frac{1}{2}, \epsilon(z)$ is positive and monotonically decreasing. Furthermore, its asymptotic expansion is $\epsilon(z)=$ $\frac{z^{-2}}{24}+\frac{z^{-3}}{24}+\ldots$ as $z \rightarrow \infty$. Thus, (B.1) becomes

$$
f_{i}(\mathbf{n}, \gamma)=\ell_{i}-\frac{v_{i}}{\gamma}\left[\ln \left(v_{i} \mathbf{d}_{i}^{\top} \mathbf{n}+n_{i}+\frac{1}{2}\right)-\ln \left(v_{i} \mathbf{d}_{i}^{\top} \mathbf{n}+\frac{1}{2}\right)+\Delta_{i}(\mathbf{n})\right],
$$

where $\Delta_{i}(\mathbf{n})=\epsilon\left(v_{i} \mathbf{d}_{i}^{\top} \mathbf{n}+1\right)-\epsilon\left(v_{i} \mathbf{d}_{i}^{\top} \mathbf{n}+n_{i}+1\right)$, is the total error due to this approximation. 
Using the Taylor expansion of the logarithm, it can be shown that for any non-negative vector $\mathbf{a}$ and $c>0, \ln \left(\mathbf{a}^{\top}\lfloor\mathbf{x}\rceil+c\right)-\ln \left(\mathbf{a}^{\top} \mathbf{x}+c\right)$ is equal to

$$
\sum_{k=1}^{\infty} \frac{(-1)^{k+1}}{k}\left[\frac{\mathbf{a}^{\top}(\lfloor\mathbf{x}\rceil-\mathbf{x})}{\mathbf{a}^{\top} \mathbf{x}+c}\right]^{k} \sim O\left(\frac{1}{\mathbf{a}^{\top}\lfloor\mathbf{x}\rceil+1}\right) .
$$

Thus, we can rewrite (B.2) as

$$
f_{i}(\mathbf{n}, \gamma)=\ell_{i}-\frac{v_{i}}{\gamma}\left[\ln \left(v_{i} \mathbf{d}_{i}^{\top} \mathbf{x}+x_{i}+\frac{1}{2}\right)-\ln \left(v_{i} \mathbf{d}_{i}^{\top} \mathbf{x}+\frac{1}{2}\right)+\Delta_{i}(\mathbf{n})+\eta_{i}(\mathbf{x})\right],
$$

where $\eta_{i}(\mathbf{x})$ is the rounding error.

Now, consider the equation

$$
0=\ell_{i}-\frac{v_{i}}{\gamma}\left[\ln \left(v_{i} \mathbf{d}_{i}^{\top} \mathbf{x}+x_{i}+\frac{1}{2}\right)-\ln \left(v_{i} \mathbf{d}_{i}^{\top} \mathbf{x}+\frac{1}{2}\right)\right]
$$

Using logarithm identities, this becomes

$$
\gamma \frac{\ell_{i}}{v_{i}}=\ln \left(\frac{v_{i} \mathbf{d}_{i}^{\top} \mathbf{x}+x_{i}+\frac{1}{2}}{v_{i} \mathbf{d}_{i}^{\top} \mathbf{x}+\frac{1}{2}}\right),
$$

which can be written as $x_{i}-\left(e^{\gamma \ell_{i} / v_{i}}-1\right) v_{i} \mathbf{d}_{i}^{\top} \mathbf{x}=\frac{1}{2}\left(e^{\gamma \ell_{i} / v_{i}}-1\right)$. Thus, we obtain a system of $m$ equations,

$$
\left[\mathbb{I}-\left(K_{\gamma}-\mathbb{I}\right) V_{1} D\right] \mathbf{x}=\frac{1}{2}\left(K_{\gamma}-\mathbb{I}\right) \mathbf{1} .
$$

Substituting the expression for $D$ into (B.5) and rearranging terms we obtain (20), and thus, (B.4) is satisfied for the considered $\mathbf{x}$ and $\gamma$. Substituting (B.4) into (B.3), we get

$$
\left|f_{i}(\mathbf{n}, \gamma)\right|=\frac{v_{i}}{\gamma}\left|\Delta_{i}(\mathbf{n})+\eta_{i}(\mathbf{x})\right| .
$$

Note that $|\Delta(\mathbf{n})|<\epsilon\left(v_{i} \mathbf{d}_{i}^{\top} \mathbf{n}+1\right)$, and so $\Delta(\mathbf{n}) \sim O\left[\left(v_{i} \mathbf{d}_{i}^{\top} \mathbf{n}+1\right)^{-2}\right]$. Additionally, $\eta_{i}(\mathbf{x}) \sim O\left[\left(v_{i} \mathbf{d}_{i}^{\top} \mathbf{n}+1\right)^{-1}\right]$.

Thus $\left|f_{i}(\mathbf{n}, \gamma)\right| \sim O\left(\left(v_{i} \mathbf{d}_{i}^{\top} \mathbf{n}+1\right)^{-1}\right)$, completing the proof.

\section{Appendix C. Proof of Theorem 4}

Let $M=\left(K_{\gamma}-\mathbb{I}\right)^{-1} K_{\gamma}-V_{1}\left(\mathbb{I}-R_{11}\right)^{-1} V_{1}^{-1}$. Assume that $M$ is invertible. Using Woodbury's identity ${ }^{4}$, we can write $M^{-1}$ as

$$
\left(\mathbb{I}-K_{\gamma}^{-1}\right)+\left(\mathbb{I}-K_{\gamma}^{-1}\right) V_{1} K_{\gamma}\left(\mathbb{I}-R_{11} K_{\gamma}\right)^{-1} V_{1}^{-1}\left(\mathbb{I}-K_{\gamma}^{-1}\right) .
$$

Therefore, $M$ is invertible only if $\mathbb{I}-R_{11} K_{\gamma}$ is invertible.

$$
{ }^{4}(A+U C V)^{-1}=A^{-1}-A^{-1} U\left(C^{-1}+V A^{-1} U\right)^{-1} V A^{-1} .
$$


Let $\lambda\left(R_{11}\right)$ denote the spectral radius of $R_{11}$. It can be shown that $\mathbb{I}-R_{11}$ is an invertible M-matrix (see Rodriguez-Vega et al. (2019)), and thus, $\lambda\left(R_{11}\right)<$ 1. For sufficiently small $\gamma, K_{\gamma}$ can be made arbitrarily close to $\mathbb{I}$, such that $\lambda\left(R_{11} K_{\gamma}\right)<1$.

Let $\gamma_{\max }$ be such that $\lambda\left(R_{11} K_{\gamma_{\max }}\right)=1$. Thus, for every $\gamma<\gamma_{\max }, \mathbb{I}-$ $R_{11} K_{\gamma}$ is an invertible M-matrix such that

$$
\left(\mathbb{I}-R_{11} K_{\gamma}\right)^{-1}=\mathbb{I}+\sum_{k=1}^{\infty}\left(R_{11} K_{\gamma}\right)^{k} .
$$

As $\gamma \rightarrow \gamma_{\max }$, the nonzero elements of $\left(R_{11} K_{\gamma}\right)^{k}$ increase exponentially. For $\gamma=\gamma_{\max }$, the sum diverges and the matrix is not invertible. Finally, for $0<$ $\gamma<\gamma_{\max },\left(\mathbb{I}-R_{11} K_{\gamma}\right)^{-1}$ and $\left(\mathbb{I}-K_{\gamma}^{-1}\right)$ are non-negative, which implies that $M^{-1}$ is non-negative.

\section{Appendix D. Proof of Theorem 5}

Because of the independence between the vectors $\mathbf{u}$ and $\mathbf{v}$, we can rewrite the desired probability in terms of the conditional probability

$$
P_{\mathbf{u}, \mathbf{v}}\left(\left|\mathbf{u}^{\top} \mathbf{v}\right|<\epsilon\right)=P_{\mathbf{u}}\left(\left|\mathbf{u}^{\top} \mathbf{v}\right|<\epsilon \mid \mathbf{v}=\mathbf{y}\right)
$$

where $\mathbf{y} \in \mathbb{S}^{m}$ is any arbitrary vector.

Consider the set $\mathcal{P}_{\epsilon}=\left\{\mathbf{x} \in \mathbf{S}^{m}:\left|\mathbf{x}^{\top} \mathbf{y}\right|<\epsilon\right\}$. This can be visualized in the unit $m$-sphere as all points within a distance $\epsilon$ from the equator, where the pole is $\mathbf{y}$. Let $\mathcal{A}=\left\{\mathbf{x} \in \mathbb{S}^{m}: \mathbf{x}^{\top} \mathbf{y}>0\right\}$ be the hemisphere of $\mathbb{S}^{m}$ closest to $\mathbf{y}$, and $\mathcal{B}=\left\{\mathbf{x} \in \mathbb{S}^{m}: \mathbf{x}^{\top} \mathbf{y} \leq 0\right\}$ be the opposite hemisphere. Note that $P_{\mathbf{u}}(\mathcal{A})=P_{\mathbf{u}}(\mathcal{B})=1 / 2$.

Define the $\epsilon$-extension $\mathcal{A}_{\epsilon}=\left\{\mathbf{x} \in \mathbb{S}^{m}: d(\mathbf{x}, \mathcal{A})<\epsilon\right\}$, and $\mathcal{B}_{\epsilon}$ defined analogously. It follows that $\mathcal{P}_{\epsilon}=\mathcal{A}_{\epsilon} \cap \mathcal{B}_{\epsilon}$. Due to the fact that $\mathbf{u}$ is independent from the choice of $\mathbf{y}$, the events $\mathcal{A}_{\epsilon}$ and $\mathcal{B}_{\epsilon}$ are independent, and thus

$$
P_{\mathbf{u}}\left(\mathcal{P}_{\epsilon}\right)=P_{\mathbf{u}}\left(\mathcal{A}_{\epsilon}\right) P_{\mathbf{u}}\left(\mathcal{B}_{\epsilon}\right)
$$

Because of the concentration of measure phenomenon, (see Theorem 2.3 in Ledoux (2005)), we have that

$$
\begin{aligned}
& P_{\mathbf{u}}\left(\mathcal{A}_{\epsilon}\right) \geq 1-e^{-(m-1) \epsilon^{2} / 2}, \\
& P_{\mathbf{u}}\left(\mathcal{B}_{\epsilon}\right) \geq 1-e^{-(m-1) \epsilon^{2} / 2} .
\end{aligned}
$$

Substitution of (D.2) and (D.3) into (D.1) gives

$$
P_{\mathbf{u}, \mathbf{v}}\left(\left|\mathbf{u}^{\top} \mathbf{v}\right|<\epsilon\right) \geq 1-2 e^{-(m-1) \epsilon^{2} / 2},
$$

thus, finalizing the proof. 


\section{References}

Aboudolas, K., Geroliminis, N., 2013. Perimeter and boundary flow control in multi-reservoir heterogeneous networks. Transportation Research Part B: Methodological 55, 265-281.

Abramowitz, M., Stegun, I., 1972. Handbook of Mathematical Function with Formulas, Graphs, and Mathematical Tables, 10th Edition. United States Department of Commerce, National Institute of Standards and Technology.

Barceló, J., Casas, J., 2005. Dynamic Network Simulation with AIMSUN. In: Simulation Approaches in Transportation Analysis. Springer-Verlag, New York, pp. 57-98.

Bekiaris-Liberis, N., Roncoli, C., Papageorgiou, M., sep 2016. Highway Traffic State Estimation with Mixed Connected and Conventional Vehicles. IFACPapersOnLine 49 (3), 309-314.

Bianchin, G., Pasqualetti, F., Kundu, S., jul 2019. Resilience of Traffic Networks with Partially Controlled Routing. In: 2019 American Control Conference (ACC). IEEE, pp. 2670-2675.

Burden, R. L., Faires, J. D., 1985. Numerical Analysis, 3rd Edition. Prindle, Weber \& Schmidt, Boston, MA.

Daganzo, C. F., 1994. The cell transmission model: A dynamic representation of highway traffic consistent with the hydrodynamic theory. Transportation Research Part B 28 (4), 269-287.

Daganzo, C. F., 1995. The cell transmission model, part II: Network traffic. Transportation Research Part B 29 (2), 79-93.

Fernando, T. L., Hieu Minh Trinh, Jennings, L., may 2010. Functional Observability and the Design of Minimum Order Linear Functional Observers. IEEE Transactions on Automatic Control 55 (5), 1268-1273.

Geroliminis, N., Daganzo, C. F., nov 2008. Existence of urban-scale macroscopic fundamental diagrams: Some experimental findings. Transportation Research Part B: Methodological 42 (9), 759-770.

Gu, Y., Qian, Z. S., Zhang, G., 2017. Traffic state estimation for urban road networks using a link queue model. Transportation Research Record 2623 (1), 29-39.

Haddad, J., Geroliminis, N., 2012. On the stability of traffic perimeter control in two-region urban cities. Transportation Research Part B: Methodological 46 (9), 1159-1176.

Haddad, J., Zheng, Z., 2020. Adaptive perimeter control for multi-region accumulation-based models with state delays. Transportation Research Part B: Methodological 137, 133-153. 
He, S. X., 2013. A graphical approach to identify sensor locations for link flow inference. Transportation Research Part B: Methodological 51 (516), 65-76.

Herrera, J. C., Bayen, A. M., 2010. Incorporation of Lagrangian measurements in freeway traffic state estimation. Transportation Research Part B: Methodological 44 (4), 460-481.

Jabari, S. E., 2016. Node modeling for congested urban road networks. Transportation Research Part B: Methodological 91, 229-249.

Ladino, A., Canudas-de Wit, C., Kibangou, A., Fourati, H., Rodriguez, M., 2018. Density and flow reconstruction in urban traffic networks using heterogeneous data sources. In: IEEE European Control Conference (ECC). Limassol, Ciprus, pp. 1679-1684.

Ledoux, M., 2005. The Concentration of Measure Phenomenon. Vol. 89 of Mathematical Surveys and Monographs. American Mathematical Society, Providence, Rhode Island.

Lovisari, E., Canudas-de Wit, C., Kibangou, A. Y., 2016. Density/Flow reconstruction via heterogeneous sources and Optimal Sensor Placement in road networks. Transportation Research Part C: Emerging Technologies 69, 451476.

Ng, M. W., 2012. Synergistic sensor location for link flow inference without path enumeration: A node-based approach. Transportation Research Part B: Methodological 46 (6), 781-788.

Niazi, M. U. B., Canudas-de Wit, C., Kibangou, A. Y., 2019. Average observability of large-scale network systems. 2019 18th European Control Conference (ECC) (694209), 1506-1511.

Pueboobpaphan, R., Nakatsuji, T., 2006. Real-Time Traffic State Estimation on Urban Road Network: The Application of Unscented Kalman Filter. pp. $542-547$.

Rodriguez-Vega, M., Canudas-de Wit, C., Fourati, H., 2019. Location of turning ratio and flow sensors for flow reconstruction in large traffic networks. Transportation Research Part B: Methodological 121, 21-40.

Rostami Shahrbabaki, M., Safavi, A. A., Papageorgiou, M., Setoodeh, P., Papamichail, I., jun 2020. State estimation in urban traffic networks: A twolayer approach. Transportation Research Part C: Emerging Technologies 115, 102616.

Sadamoto, T., Ishizaki, T., Imura, J. I., 2017. Average state observers for largescale network systems. IEEE Transactions on Control of Network Systems 4 (4), 761-769. 
Saedi, R., Saeedmanesh, M., Zockaie, A., Saberi, M., Geroliminis, N., Mahmassani, H. S., 2020. Estimating network travel time reliability with network partitioning. Transportation Research Part C: Emerging Technologies 112 (January), 46-61.

Seo, T., Bayen, A. M., Kusakabe, T., Asakura, Y., 2017. Traffic state estimation on highway: A comprehensive survey. Annual Reviews in Control 43, 128-151.

Seo, T., Kusakabe, T., Asakura, Y., 2015a. Estimation of flow and density using probe vehicles with spacing measurement equipment. Transportation Research Part C: Emerging Technologies 53, 134-150.

Seo, T., Kusakabe, T., Asakura, Y., 2015b. Traffic State Estimation with the Advanced Probe Vehicles Using Data Assimilation. IEEE Conference on Intelligent Transportation Systems, Proceedings, ITSC 2015-Octob, 824-830.

Sun, Y., Work, D. B., 2014. A distributed local Kalman consensus filter for traffic estimation. Proceedings of the IEEE Conference on Decision and Control 2015-Febru (February), 6484-6491.

Tampère, C. M., Immers, L. H., 2007. An extended Kalman filter application for traffic state estimation using CTM with implicit mode switching and dynamic parameters. In: IEEE Intelligent Transportation Systems Conference. Seattle, USA, pp. 209-216. 